\title{
Type III Neuregulin 1 Is Required for Multiple Forms of Excitatory Synaptic Plasticity of Mouse Cortico-Amygdala Circuits
}

\author{
Li Jiang, ${ }^{1,3}$ Jaime Emmetsberger, ${ }^{2,3}$ David A. Talmage, ${ }^{2,3}$ and Lorna W. Role ${ }^{1,3,4}$ \\ ${ }^{1}$ Department of Neurobiology and Behavior, ${ }^{2}$ Department of Pharmacological Sciences, ${ }^{3}$ Center for Nervous Systems Disorders Research, and \\ ${ }^{4}$ Neurosciences Institute, Stony Brook University, Stony Brook, New York 11794
}

\begin{abstract}
The amygdala plays an important role in the formation and storage of memories associated with emotional events. The cortical glutamatergic inputs onto pyramidal neurons in the basolateral nucleus of the amygdala (BLA) contribute to this process. As the interaction between neuregulin $1(\mathrm{Nrg} 1)$ and its ErbB receptors has been implicated in the pathological mechanisms of schizophrenia, loss of $\mathrm{Nrg} 1$ may disrupt cortical-amygdala neural circuits, resulting in altered processing of salient memories. Here we show that Nrg1 is critical in multiple forms of plasticity of cortical projections to pyramidal neurons of the BLA. The miniature EPSCs in Nrg1 heterozygous animals have a faster time constant of decay and evoked synaptic currents have a smaller NMDA/AMPA ratio than those recorded in wild-type (WT) littermates. Both high-frequency electrical stimulation of cortical inputs and $\theta$ burst stimulation combined with nicotine exposure results in long-lasting potentiation in WT animals. However, the same manipulations have little to no effect on glutamatergic synaptic plasticity in the BLA from $\mathrm{Nrg} 1$ heterozygous mice. Comparison of WT, $\mathrm{Nrg} 1$ heterozygous animals and $\boldsymbol{\alpha} 7$ nicotinic receptor heterozygous mice reveals that the sustained phase of potentiation of glutamatergic transmission after $\theta$ burst stimulation with or without nicotine only occurs in the WT mice. Together, these findings support the idea that type III Nrg1 is essential to multiple aspects of the modulation of excitatory plasticity at cortical-BLA synapses.
\end{abstract}

\section{Introduction}

Neuregulin 1 (Nrg1) and its signaling partner, the ErbB4 receptor, have been identified as leading candidate schizophrenia susceptibility genes (Stefansson et al., 2002; Harrison and Weinberger, 2005; Harrison and Law, 2006; Lu et al., 2010; Stefanis et al., 2011; Bae et al., 2012). Nrg1 signaling is important in nervous system development contributing to aspects of neuronal migration, synaptogenesis, gliogenesis, and neuron-glia communication (Taveggia et al., 2005; Lopez-Bendito et al., 2006; Quintes et al., 2010; Ting et al., 2011). In mature animals, alterations in Nrg1/ErbB signaling are manifest in aberrant peripheral myelination, and deficits in synaptic plasticity in hippocampal and cortical slices (Michailov et al., 2004; Kwon et al., 2005; Bjarnadottir et al., 2007; Li et al., 2007; Brinkmann et al., 2008; Chen et al., 2008b; Pitcher et al., 2011; Shamir et al., 2012). Likewise, Nrg1 and ErbB receptor interactions have been shown to affect the

Received June 15, 2012; revised April 18, 2013; accepted April 19, 2013.

Author contributions: L.J., J.E., D.A.T., and L.W.R. designed research; L.J. and J.E. performed research; L.J., D.A.T., and L.W.R. contributed unpublished reagents/analytic tools; L.J., J.E., D.A.T., and L.W.R. analyzed data; L.J., J.E., D.A.T., and L.W.R. wrote the paper.

This work was supported by the National Institutes of Health Grants NS29071, NSO22061, and MH087473 to L.W.R. and D.A.T., Sydney R. Baer Foundation/National Alliance for Research on Schizophrenia and Depression (L.W.R. and D.A.T.), and the McKnight Brain Disorders Foundation (L.W.R.). We thank Young-Hwan Jo for critiques of the manuscript and Catherine Waddell and Yehui Qin for technical support.

The authors declare no competing financial interests.

Correspondence should be addressed to Dr. Li Jiang, Stony Brook University, 100 Nicolls Road, Stony Brook, NY 11794. E-mail: Li.Jiang@stonybrook.edu.

DOI:10.1523/JNEUROSCI.2888-12.2013

Copyright $\odot 2013$ the authors $\quad 0270-6474 / 13 / 339655-12 \$ 15.00 / 0$ synaptic function of many neuronal transmitter receptors, including glutamate $\alpha$-amino-3-hydroxy-5-methyl-4-isoxazole propionic acid (AMPA) receptors, $N$-methyl-D-aspartate (NMDA) receptors, and nicotinic acetylcholine receptors (nAChRs) (Kwon et al., 2005; Bjarnadottir et al., 2007; Hancock et al., 2008; Zhong et al., 2008; Abe et al., 2011; Canetta et al., 2011; Pitcher et al., 2011; Ting et al., 2011; Fenster et al., 2012).

In the hippocampus and neocortex, Nrg1/ErbB4 signaling has a major impact on the formation and function of glutamatergic transmission. Nrg1 activation of ErbB4 in cortical and hippocampal interneurons induces formation of glutamatergic synapses on these interneurons (Fazzari et al., 2010; Ting et al., 2011) and acutely regulates excitatory plasticity of pyramidal neurons (Li et al., 2007; Chen et al., 2010a, 2010b; Fazzari et al., 2010; Pitcher et al., 2011; Bae et al., 2012, Fenster et al., 2012; Shamir et al., 2012).

The type III Nrg1 isoform is also an important regulator of cholinergic signaling. Nrg1 regulates both the expression of AChR genes and targeting of nAChRs to the neuronal cell surface (Sandrock et al., 1997; Yang et al., 1998; Chang and Fischbach, 2006), and the type III Nrg1 isoforms in particular are critical regulators of presynaptic targeting of $\alpha 7$-containing $\left(\alpha 7^{\star}\right)$ nAChRs (Chen et al., 2008b; Hancock et al., 2008; Zhong et al., 2008).

The possible role of Nrg1/ErbB signaling in the amygdala has not been explored. Type III Nrg1 is expressed in the basolateral nucleus of the amygdala (BLA) and in cortical neurons that project to the BLA (see below), and ErbB4 is expressed in interneu- 
rons within the BLA as well as highly expressed in the basomedial area (Shamir et al., 2012). The BLA receives extensive cholinergic input from the nucleus basalis of Meynert (Carlsen et al., 1985; Wenk, 1997; Schafer et al., 1998; Muller et al., 2011). Both $\alpha 7^{*}$ and non $\alpha 7^{\star}$ nAChRs have been shown to contribute to presynaptic modulation of cortical-BLA synapses by exogenously applied nicotine (Jiang and Role, 2008).

To gain insight into the functional significance of $\mathrm{Nrg} 1 / \mathrm{ErbB}$ signaling in the BLA, we investigated whether heterozygous deletion of type III Nrg1 alters cortical-BLA transmission and/or changes the efficacy of cholinergic modulation of these circuits. We found that heterozygous deletion of type III Nrg 1 blocked the induction of LTP at cortical-BLA synapses, whether activated by patterned stimulation alone or by concurrent electrical stimulation paired with nicotine exposure. Parallel studies in mice heterozygous for $\alpha 7^{\star}$ nAChR revealed a direct phenocopy of plasticity deficits in the type III $\mathrm{Nrg} 1$ heterozygous mice, and type III Nrg1 heterozygotes have reduced $\alpha 7^{\star}$ nAChRs along projections within the BLA. Hence, both type III Nrg1 and $\alpha 7$ containing nAChRs appear to be critical in inducing LTP of glutamatergic transmission in BLA.

\section{Materials and Methods}

All mouse care and experimental procedures were approved by the Institutional Animal Care Research Advisory Committee of the State University of New York at Stony Brook. Mice of either sex used in these experiments include wild-type (WT) (C57 BL6), Nrg1 ${ }^{\text {Lwrtm1+/- }}$ (Wolpowitz et al., 2000) and Chrna $7^{\text {Baytm1 }}\left(\alpha 7^{+/-}\right.$or $\left.\alpha 7^{-1-}\right)$ (The Jackson Laboratory). Both mutant lines are maintained on a pure C57 BL6 background. All experiments were performed before weaning.

Slice preparation. Coronal brain slices were prepared from WT mice or from $\mathrm{Nrg} 1$ or $\alpha 7$ mutant mice at postnatal age 14-20 d. Animals were anesthetized with a mixture of ketamine and xylazine (100 mg ketamine and $6 \mathrm{mg}$ xylazine/kg body weight injected i.p.). After decapitation, the brain was transferred quickly into a sucrose-based solution bubbled with $95 \% \mathrm{O}_{2}$ and $5 \% \mathrm{CO}_{2}$ and maintained at $\sim 3^{\circ} \mathrm{C}$. This solution contained the following (in $\mathrm{mM}$ ): sucrose $230, \mathrm{KCl} 2.5, \mathrm{MgSO}_{4} 10, \mathrm{CaCl}_{2} 0.5$, $\mathrm{NaH}_{2} \mathrm{PO}_{4} 1.25, \mathrm{NaHCO}_{3} 26$, and glucose 10. Coronal brain slices (300 $\mu \mathrm{m}$ ) were prepared using a Leica VT1000S vibratome (Leica). Slices were equilibrated with an oxygenated artificial CSF (aCSF) at room temperature $\left(24-26^{\circ} \mathrm{C}\right)$ for at least $1 \mathrm{~h}$ before transfer to the recording chamber. The slices were continuously superfused with aCSF at a rate of $2 \mathrm{ml} / \mathrm{min}$ containing the following (in $\mathrm{mM}$ ): $\mathrm{NaCl} 126, \mathrm{KCl} 2.5, \mathrm{NaH}_{2} \mathrm{PO}_{4} 1.25$, $\mathrm{NaHCO}_{3} 26, \mathrm{CaCl}_{2} 2, \mathrm{MgCl}_{2}$ 2, and glucose 10 bubbled with $95 \% \mathrm{O}_{2}$ and $5 \% \mathrm{CO}_{2}$ at room temperature.

Electrophysiological recordings. Brain slices were placed on the stage of an upright, infrared-differential interference contrast microscope (BX51WI, Olympus Optical). BLA pyramidal neurons were visualized with a $40 \times$ water-immersion objective by infrared microscopy (4915 camera, COHU). Patch electrodes with a resistance of $4-6 \mathrm{M} \Omega$ were pulled with a laser-based micropipette puller (P-2000, Sutter Instrument). Signals were recorded with a Multi Clamp 700A amplifier and pClamp10 software (Molecular Devices). The pipette solution contains the following (in mM): $130 \mathrm{~K}$-gluconate, $2 \mathrm{KCl}$, $2 \mathrm{MgCl}_{2}, 10$ HEPES, 0.5 EGTA, 1 ATP, and $0.2 \mathrm{GTP}, \mathrm{pH}$ 7.3. To examine the voltage dependence of the evoked PSCs, $130 \mathrm{~mm}$ Cs-methane-sulfonate was used instead of K-gluconate. All recordings included in this study are from rapidly activating and strongly accommodating pyramidal neurons (Jiang and Role, 2008). The following criteria were applied for inclusion of recorded cells in the study; failure to meet all of these criteria resulted in exclusion from the sample population: (1) seal resistances maintained throughout the recording period at $>5 \mathrm{G} \Omega$; (2) holding current in whole-cell clamp configuration remained within $10 \%$ of the initial value and was $\leq 100 \mathrm{pA}$; and (3) series resistance; $R_{s}$ measured every 10 min throughout the course of the experiment remained stable (i.e., $<10 \%$ change from initial value).

To examine transmission at cortical-BLA inputs, EPSCs were evoked by field stimulation with a concentric bipolar stimulation electrode (FHC) placed in the external capsule. A $0.1 \mathrm{~Hz}$ single stimulation (dura- tion, $1 \mathrm{~ms}$; intensity, $10-30 \mu \mathrm{A}$ ) was delivered via the Master- 8 stimulator (A.M.P.I.). For minimal stimulation, the stimulation strength was adjusted to trigger EPSCs in response to stimulation in $\sim 50 \%$ of the trials, which was typically equivalent to activation at $\sim 10-15 \%$ of the maximum response amplitude. In some experiments, a stronger stimulation was used (20-30\% of maximal response as in Tsvetkov et al., 2004). High-frequency stimulation (HFS, $1 \mathrm{~s}$ at $100 \mathrm{~Hz}$ ) $2 \mathrm{~ms}$ pulses were delivered 5 times with $10 \mathrm{~s}$ interval. The $\theta$ burst stimulation pattern (TBS) used delivered four $2 \mathrm{~ms}$ pulses of depolarization at $50 \mathrm{~Hz}$, repeated 10 times at a $5 \mathrm{~Hz}$ interbust interval. Spontaneous EPSCs in BLA pyramidal neurons were monitored in a voltage-clamp configuration at a holding potential of $-60 \mathrm{mV}$. Bicuculline $(10 \mu \mathrm{M})$ was continuously present in aCSF to isolate glutamatergic synaptic transmission. Miniature EPS currents were recorded in the presence of $1 \mu \mathrm{M}$ TTX. Data were filtered at $2 \mathrm{kHz}$ by Multi Clamp 700A and analyzed using Clampfit10 (Axon Instruments) and Mini Analysis 6.0 (Synaptosoft).

Statistical analyses were performed using nonparametric statistical tests as the majority of electrophysiological measures were not normally distributed. Tests included the Kolmogorov-Smirnov (KS), MannWhitney (MW), and paired-sample Wilcoxon signed rank test with Origin 9.0 (Originlab). The median values were reported from the entire population tested. Data were considered significantly different when $p<$ 0.05 . The study is based on $>200$ recordings from 81 mice, unless otherwise noted; $n$ values reported are numbers of recordings.

Nicotine delivery and doses. Nicotine (500 nM) was applied with a picospritzer (Parker Instrumentation). Pressure (5-10 psi) was applied for $1 \mathrm{~min}$ to a $1-2 \mu \mathrm{m}$ diameter patch pipette that was positioned $\sim 15 \mu \mathrm{m}$ from the soma of the recorded neurons. All experiments with acute nicotine were performed in the presence of $200 \mathrm{~nm}$ atropine to preclude muscarinic receptor activation.

Anterograde tracing. P21 mice were anesthetized using isoflurane, and holes were drilled in the skull using a $0.7 \mathrm{~mm}$ drill bit (Fine Science Tools) $1.54 \mathrm{~mm}$ anterior and $0.45 \mathrm{~mm}$ lateral to bregma. A 25 gauge Hamilton syringe was then inserted at a depth of $1.5 \mathrm{~mm}$ from the dural surface and $1 \mu \mathrm{l}$ of $10 \%$ biotin dextran amine (BDA; Invitrogen) conjugate was injected manually over $3 \mathrm{~min}$, and the needle was withdrawn after an additional 2 min to prevent reflux. The skin above the skull was closed with tissue adhesive (3M vetbond), and the animals were placed on a heating pad until they recovered from the anesthesia. The animals were administered analgesic, every day until death $5 \mathrm{~d}$ after injection via transcardial perfusion.

Immunofluorescence. For immunofluorescent analysis, P21-P28 mice were transcardially perfused with $4 \%$ PFA in PBS. After perfusion the brains were isolated, postfixed with $4 \%$ PFA, cryoprotected in 30\% sucrose for $2 \mathrm{~d}$, and stored at $-80^{\circ} \mathrm{C}$ in OCT until sectioned. For $\alpha 7 \mathrm{nAChR}$ surface expression, frozen sections $(15 \mu \mathrm{m})$ were blocked for $1 \mathrm{~h}$ with $5 \%$ normal donkey serum and incubated for $1 \mathrm{~h}$ with $\alpha$-bungarotoxin conjugated to biotin XX ( $\alpha$-BgTx-biotin XX; 1:1000; Invitrogen). Antigen retrieval was performed to detect CTIP-2 (Abcam, 1:500) by incubating sections in sodium citrate buffer, $\mathrm{pH} 6$, for $15 \mathrm{~min}$ at $100^{\circ} \mathrm{C}$ followed by blocking step, as stated below. To detect type III Nrg1, BDA and CTIP-2 sections $(15 \mu \mathrm{m})$ were blocked and permeabilized with 5\% normal donkey serum containing $0.1 \%$ Triton X-100 (Sigma) for $1 \mathrm{~h}$ at room temperature followed by overnight incubation at $4^{\circ} \mathrm{C}$ with sheep anti-CRD (1:100; for full description and characterization of this antibody, see Yang et al., 1998, Chen et al., 2010a, Hancock et al., 2011) or a $1 \mathrm{~h}$ incubation with streptavidin 680 to detect type III Nrg1 and BDA, respectively. After washes, total $\alpha 7^{\star} \mathrm{nAChR}$ expression was detected by incubating the slices for $1 \mathrm{~h}$ with $\alpha$-BgTx-biotin XX (Invitrogen) or $\alpha$-BgTx conjugated to Alexa488 ( $\alpha$-BgTx-488; Invitrogen) at a 1:1000 dilution. Sections were washed and incubated for $1 \mathrm{~h}$ at room temperature with respective secondary antibodies.

\section{Results}

Previously, we demonstrated that presynaptic nAChRs play an important role in modulating cortical-amygdala synapses (Jiang and Role, 2008) and that type III Nrg1 is a key regulator of presynaptic targeting of $\alpha 7^{\star} \mathrm{nAChRs}$ in neurons in culture (Hancock 
et al., 2008; Zhong et al., 2008). In this study, we sought to determine whether plasticity and presynaptic nicotinic modulation of cortical-BLA synapses are affected in mice heterozygous for a mutation in the type III Nrg1 gene.

The results presented are from $>200$ total recordings from 81 mice. Because the type III Nrg1 knock-out mice die at birth, only heterozygotes for type III Nrg1 (HET) were used in the electrophysiological studies. Only records that passed all recording criteria as outlined in Materials and Methods were included in the analyses.

Type III Nrg1 is expressed in cortical areas that innervate the BLA: partial deletion of Nrg1 alters the kinetics of miniature EPSCs (mEPSCs)

We examined the expression of type III Nrg1 in the cingulate cortex. Immunostaining with specific antibodies against type III Nrg1 (Yang et al., 1998, Chen et al., 2010a, 2010b; Hancock et al., 2011) and CTIP-2, a transcription factor expressed by deep layer subcortical projection neurons (Arlotta et al., 2005; Molyneaux et al., 2005; Chen et al., 2008a; McKenna et al., 2011) revealed type III Nrg1 expression in cortical pyramidal neurons (Fig. 1A) and demonstrated a pronounced reduction in type III Nrg1 expression in these same type of cortical neurons from type III NrgI heterozygous animals (Fig. $1 B$ ).

As Nrg1 has been reported to regulate the levels and function of many synaptic receptors, including AMPA- and NMDA-type glutamate receptors, we began our electrophysiological analyses by assessing the profile of baseline synaptic activity in the BLA, comparing WT mice with mice that are heterozygous for type III Nrg1 (HET). To compare miniature (TTX-resistant) glutamatergic synaptic currents in BLA of WT versus Nrg1 HET mice, we included both bicuculline $(10 \mu \mathrm{M})$ and TTX $(1 \mu \mathrm{M})$ in the aCSF. Analysis of a large number of TTX-resistant events recorded from both WT and Nrg1 HET revealed no difference in mEPSC frequency (Fig. $1 C, D ; p=0.87, \mathrm{KS}$ test, WT, $n=14$, HET, $n=8$; inset, box plot of mEPSC frequency, $p=0.59$, MW test). Although the amplitudes of mEPSCs in the BLA of Nrg1 HET mice were somewhat larger than those recorded in WT littermates, the difference does not reach statistical significance (Fig. $1 E ; p=$ 0.12 , KS test, inset, box plot of amplitude data, $p=0.32$, MW test). In contrast, comparison of the decay kinetics of the miniature synaptic currents revealed a significantly faster time constant in the Nrg1 HET than in WT, consistent with briefer open time kinetics of the underlying glutamate gated channels (Fig. $1 F, G$; $p=0.02$, MW test, WT, $n=14$; HET, $n=8$ ).

\section{Differences in evoked glutamatergic transmission at} cortical-BLA synapses of WT versus type III Nrg1 HET mice Although the baseline glutamatergic transmission appeared similar between WT and Nrg1 HET with respect to mEPSC frequency and amplitude, the differences in the kinetic profile of the mEPSCs prompted us to examine the potential effect of deletion of one allele of $\mathrm{Nrgl}$ on evoked glutamatergic transmission in more detail. To assess the relative contribution of AMPA versus NMDA receptor types to evoked glutamatergic synaptic transmission via cortical inputs, we recorded synaptic currents over a range of holding potentials (Fig. $2 A$; from $-90 \mathrm{mV}$ to $+50 \mathrm{mV}$, step $=$ $+20 \mathrm{mV}$, the resting membrane potential holding at $-60 \mathrm{mV}$ ) while stimulating the external capsule fibers. The NMDA to AMPA ratio was calculated using the amplitude of NMDA synaptic currents holding at $+50 \mathrm{mV}$ divided by the amplitude of AMPA synaptic currents holding at $-70 \mathrm{mV}$. The synaptic current NMDA to AMPA ratio in the WT mice is significantly higher
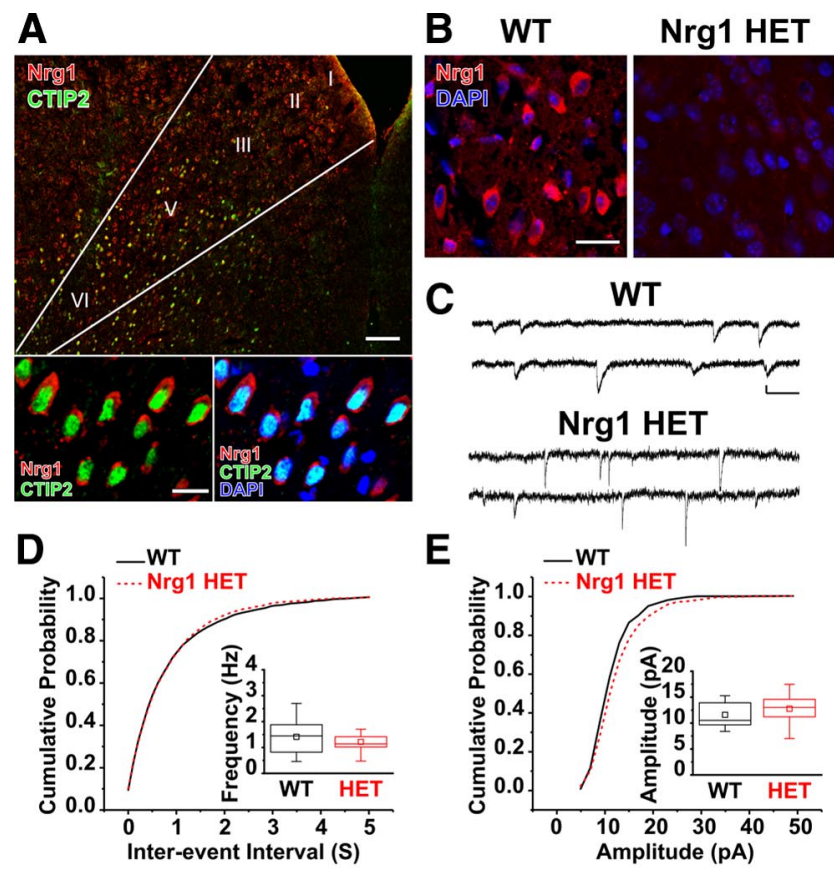

E
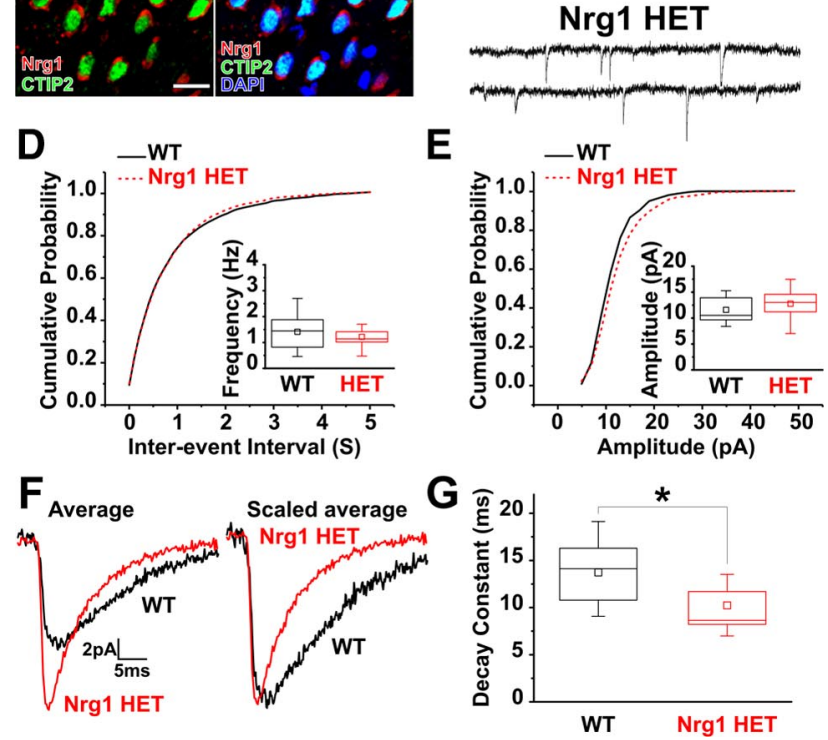

Figure 1. Nrg1 expression in cingulate cortex-BLA circuit: heterozygous deletion of type III $\mathrm{Nrg} 1$ alters properties of miniature EPSCs in BLA pyramidal neurons. $A$, Representative confocal image of Nrg-1 (red) and CTIP-2 expression in the cingulate cortex. Scale bar, $100 \mu \mathrm{m}$. Left inset, CTIP-2-expressing subcortical projection neurons (green) express type III Nrg1 in layer V of the cingulate. Right inset, The left inset merged with DAPI (dark blue) CTIP-2-positive nuclei (cyan) are pyramidal neuron; (TIP-2-negative nuclei (dark blue) are interneurons. Scale bar, 20 $\mu \mathrm{m} . \boldsymbol{B}$, Immunofluorescent micrographs of cingulate cortex labeled with type III Nrg 1 antibody (red) and DAPI (blue) in WT (left) and in the type III Nrg1 (+/-) mouse. Scale bar, $30 \mu \mathrm{m}$. $\boldsymbol{C}$, Top, Sample traces of mEPSCs recorded in a BLA pyramidal neuron in WT acute slice at a holding potential of $-60 \mathrm{mV}$ (1 $\mu \mathrm{m}$ TTX, $10 \mu \mathrm{m}$ bicuculline, and $200 \mathrm{~nm}$ atropine). Calibration: 5 pA, 100 ms. D, Bottom, Sample traces of mEPSCs recorded in a comparable BLA pyramidal neuron in an acute slice prepared from a $\mathrm{Nrg} 1 \mathrm{HET}$ recording conditions in $\boldsymbol{C}$ are otherwise identical. $\boldsymbol{D}$, Cumulative distribution of the mEPSCs interevent interval times from 22 separate experiments in WT $(n=14)$ and $\operatorname{Nrg} 1$ HET $(n=8)$ neurons; acute slice with recording conditions as in $\boldsymbol{D}$. There is no significant difference in the interevent intervals, indicating that WT and Nrg1 HET BLA neurons have similar mEPSC frequency (solid black line, WT; dotted red line, Nrg1 HET; $p=$ $0.87, \mathrm{KS}$ test). Inset, Box plot of mEPSC frequencies from the population data ( $p=0.59, \mathrm{MW}$ test). The horizontal lines in each box (starting from the lower bar) indicate the range of the data that fall within the 25 th, 50 th, and 75 th percentile values, respectively. The whiskers indicate the 5 th and 95 th percentile values. The square symbol within the box represents the mean of the population. $\boldsymbol{E}$, Cumulative distribution of the mEPSC amplitudes from WT and Nrg1 HET animals. The cumulative distribution of mEPSC amplitudes shows that $\mathrm{Nrg} 1 \mathrm{HET}$ animals have somewhat larger amplitudes, although this difference was not statistically significant (solid black line, WT; dotted red line, Nrg1 HET; WT, $n=14 ; \mathrm{HET}, n=8 ; p=0.12$, KS test). Inset, Box plot of mEPSC amplitudes from the population data ( $p=0.32$, MW test). $\boldsymbol{F}$, Left, Digital averaged $m E P S C$ recorded in representative WT BLA pyramidal neuron (black) and a representative Nrg1 HET neuron (red; number of events averaged: WT, $n=78 ; \mathrm{HET}, n=95$ ). Nrg1 HET $\mathrm{mEPSC}$ has somewhat larger amplitude and significantly faster time constant of decay than WT. Calibration: 2 pA, 5 ms. F, Right, averaged WT trace was scaled to the same amplitude as Nrg1 HET for comparison of decay time course. G, Pooled tau decay time constant (single exponential fit) for WT and Nrg1 HET mEPSCs. The decay time constant for Nrg1 HET mEPSC is significantly faster than that of WT (tau WT $=13.7 \pm 0.8 \mathrm{~ms}, n=14$ neurons; 1289 events vs tau HET, red; $10.2 \pm 1.2 \mathrm{~ms}, n=8$ neurons, 846 events; $p=0.02$, MW test). ${ }^{*} p<0.05$. 
than it is in the Nrg1 HET mice (Fig. 2B; $p=0.038$, MW test; WT, $n=7$; and HET, $n=6)$. Examination of the I versus V relationship for each of the underlying glutamate receptor-mediated currents was then performed (Fig. 2C,D). This analysis revealed that the NMDA receptor-mediated synaptic currents were not significantly different in Nrg1 HET and WT BLA pyramidal neurons, with the exception of a single holding potential (Fig. $2 C$; at $\mathrm{V}_{\mathrm{h}}-90 \mathrm{mV}, p=$ $0.35 ;-70 \mathrm{mV}, p=0.83 ;-50 \mathrm{mV}, p=1$; $-30 \mathrm{mV}, p=0.13 ;-10 \mathrm{mV}, p=0.01 ;+10$ $\mathrm{mV}, p=0.43 ;+30 \mathrm{mV}, p=0.94 ;+50 \mathrm{mV}$, $p=0.94$; MW test). However, such studies also revealed that the amplitude of AMPA receptor-mediated synaptic currents was significantly greater in $\mathrm{Nrg} 1$ HET compared with WT mice at all hyperpolarizing holding potentials (Fig. 2D; $\mathrm{WT}=7, \mathrm{HET}=6$ at $\mathrm{V}_{\mathrm{h}}-90 \mathrm{mV}, p=0.004$; $-70 \mathrm{mV}, p=0.01 ;-50 \mathrm{mV}, p=0.005$; $-30 \mathrm{mV}, p=0.015 ;-10 \mathrm{mV}, p=0.007 ;+10$ $\mathrm{mV}, p=0.70 ;+30 \mathrm{mV}, p=0.25 ;+50$ $\mathrm{mV}, p=0.12$; MW test).

We next sought to assess whether the observed differences in the AMPA receptormediated synaptic currents in WT versus Nrg1 HET mice might alter the overall input versus output relationship of cortical-BLA synapses in WT compared with Nrg1 HET. To examine the input/output relationship, we increased the stimulation intensity from 10 to $200 \mu \mathrm{A}$ (by $10 \mu \mathrm{A}$ per step) and measured the amplitude of the evoked synaptic currents in both WT and Nrg1 HET animals. The points at which the response was $10 \%$ and $90 \%$ of the maximum amplitude are compared by genotype in Figure 2E, F. At lower stimulation, the evoked synaptic currents are equivalent in WT and Nrg1 HET (Fig. 2E, F; low stimulation, $p=0.89$, MW test; WT, $n=10$; HET, $n=8$ ). With increased stimulation intensity, the amplitude of the evoked currents in the Nrg1 HET animals was somewhat larger than in WT siblings, although the trend is not statistically significant (Fig. 2E, F; high stimulation, $p=0.56$, MW test). Together, these data are consistent with the idea that, whereas the net postsynaptic responses are not significantly different, the relative contribution of the postsynaptic AMPAR- versus NMDAR-mediated responses in Nrg1 HET mice are greater than that of the WT.

\section{High-frequency burst stimulation of cortical inputs elicits LTP at cortical-BLA synapses in WT, but not in type III Nrg1 HET, mice}

Prior studies of evoked synaptic transmission have focused on the cortical projections to pyramidal neurons in the BLA
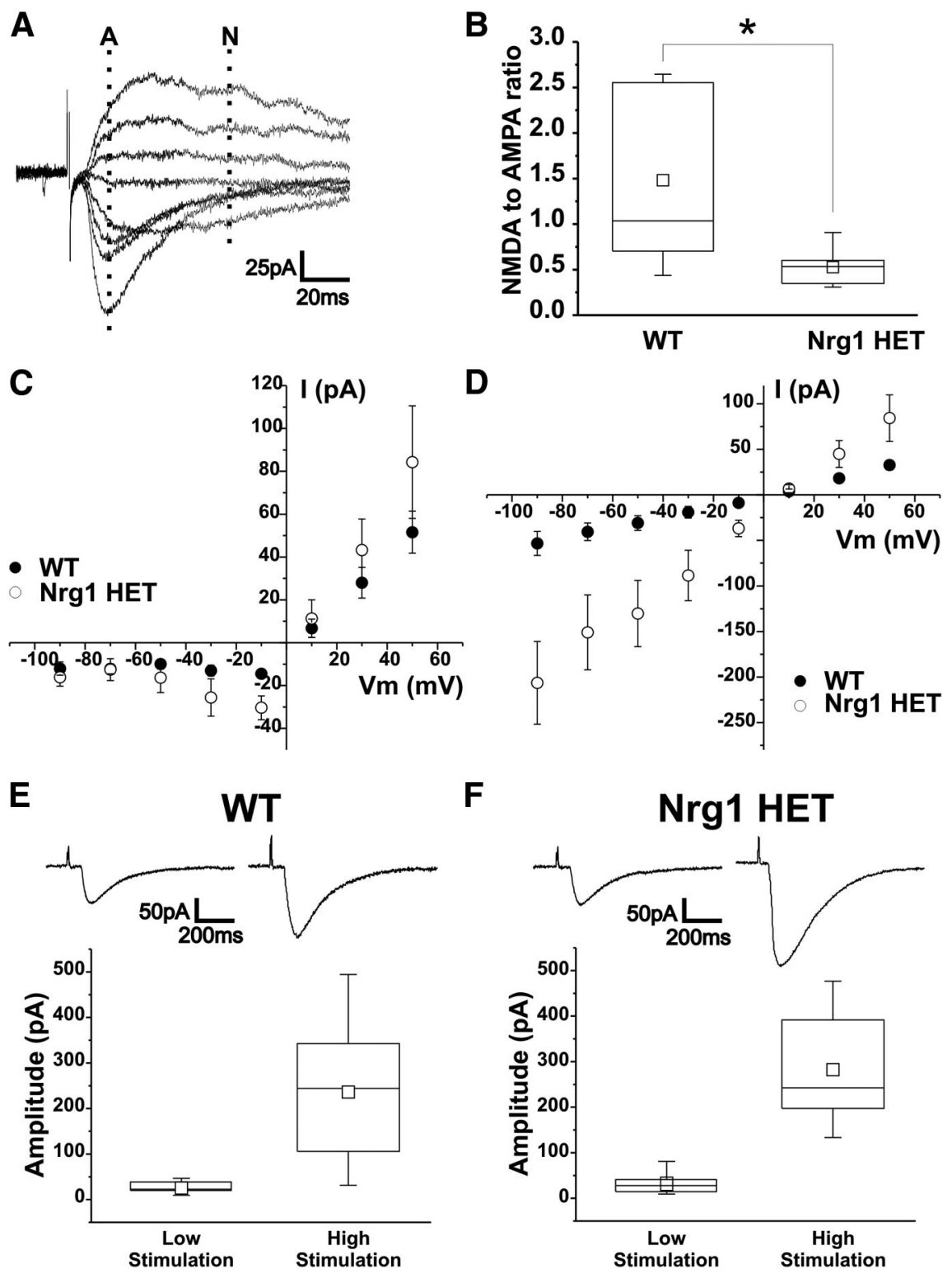

Figure 2. Heterozygous deletion of type III Nrg1 alters the properties of evoked EPSCS at cortical-BLA pyramidal neuron synapses. $A$, Sample traces of evoked EPSCs from cortical-BLA inputs with a $20 \mathrm{mV}$ step depolarization from $-90 \mathrm{mV}$ to $+50 \mathrm{mV}$ $\left(V_{h}=-60 \mathrm{mV}\right)$. The amplitude of AMPA receptor-mediated currents was measured at the peak (indicated by the dotted line labeled A). The amplitude of NMDA receptor-mediated currents was measured $50 \mathrm{~ms}$ after the EPSC peak (indicated by the dotted line labeled N). Calibration: $25 \mathrm{pA}, 20 \mathrm{~ms}$. B, NMDA to AMPA ratio for WT versus Nrg 1 HET. The NMDA to AMPA ratio was calculated as the amplitude of the NMDA receptor-mediated component measured at $V_{h}=+50 \mathrm{mV}$ divided by the amplitude of the AMPA receptor component at $\mathrm{V}_{\mathrm{h}}=-70 \mathrm{mV}$. The WT NMDA to AMPA ratio is significantly higher than that recorded in Nrg 1 HET animals (WT: $1.48 \pm 0.35, n=7$; HET: $0.53 \pm 0.04, n=6 ; p=0.04$, MW test). ${ }^{*} p<0.05$. C, Plots of I versus V relationship for NMDA-receptor-mediated component of cortical-BLA evoked EPSCS. The amplitude of the NMDA component was measured in $\boldsymbol{A}$ with steps from the holding potential as indicated. There is no significant difference between the NMDA I versus $V$ for WT and Nrg 1 HET animals at all but one holding potential (WT, $n=7$; HET, $n=6$; at $\mathrm{V}_{\mathrm{h}}-90 \mathrm{mV}, p=0.35 ;-70 \mathrm{mV}, p=0.83 ;-50 \mathrm{mV}, p=$ $1 ;-30 \mathrm{mV}, p=0.13 ;-10 \mathrm{mV}, p=0.01 ;+10 \mathrm{mV}, p=0.43 ;+30 \mathrm{mV}, p=0.94 ;+50 \mathrm{mV}, p=0.94 ;$ MW test). D, Plots of I versus $V$ relationship for AMPA-receptor-mediated component of cortical-BLA evoked EPSCs. The amplitude of the AMPA component was measured as indicated in $\boldsymbol{A}$ with steps in the holding potential. $\mathrm{Nrg} 1 \mathrm{HET}$ animals have significantly larger amplitude of AMPA receptor-mediated currents than WT at comparable hyperpolarized, but not at depolarized, holding potentials (WT, $n=7$; HET, $n=6$; at V $\mathrm{h}_{\mathrm{h}}-90 \mathrm{mV}, p=0.004 ;-70 \mathrm{mV}, p=0.01 ;-50 \mathrm{mV}, p=0.005 ;-30 \mathrm{mV}, p=$ $0.015 ;-10 \mathrm{mV}, p=0.007 ;+10 \mathrm{mV}, p=0.7 ;+30 \mathrm{mV}, p=0.25 ;+50 \mathrm{mV}, p=0.12 ;$ MW test). $\boldsymbol{E}$, Examination of input/output characteristics of evoked EPSCs at WT versus HET cortical-BLA synapses. Top, Evoked EPSC traces from cortical-BLA synapse in WT; representative EPSCs at 10\% versus $90 \%$ of maximal stimulation. Calibration: $50 \mathrm{pA}, 200 \mathrm{~ms}$. Bottom, Pooled data of responses to $10 \%$ (left) versus $90 \%$ (right) stimulation intensity in WT ( $n=10)$. $F$, Evoked EPSCS recorded in Nrg1 HET BLA ( $n=8)$ at 10\% and $90 \%$ stimulation intensity. There are no statistically significant differences between WT and Nrg 1 HET at either the lower (WT, $n=10 ; \mathrm{HET}, n=8 ; p=0.89$, MW test) or higher stimulation intensities $(p=0.56, \mathrm{MW}$ test), although a trend toward somewhat larger evoked EPSCs at higher stimulation intensities in the type III Nrg1 HET is noted. Calibration: 50 pA, 200 ms. 


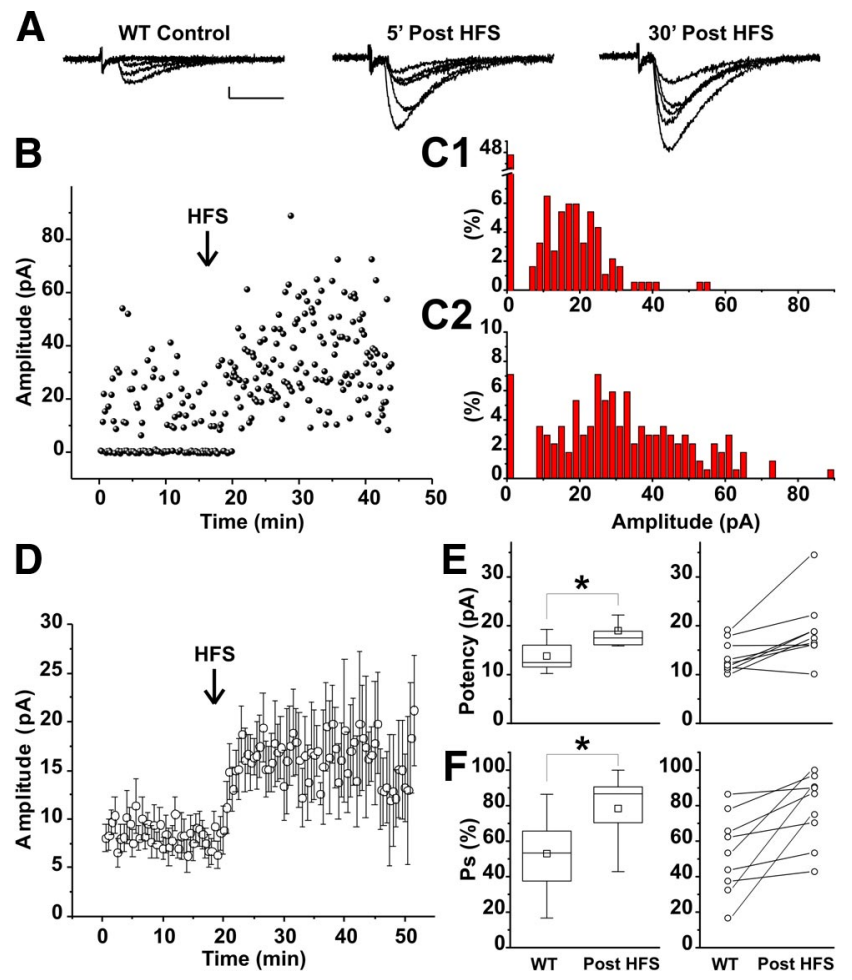

Figure 3. High-frequency burst stimulation reliably elicits LTP in cortical-BLA circuits of WT mice. A, Evoked EPSCs during control conditions $(0.1 \mathrm{~Hz}$, minimum stimulation conditions $\sim 50 \%$ failures, EPSC amplitude $\sim 10-15 \%$ maximum response amplitude), and at 5 min and at $30 \mathrm{~min}$ after HFS burst $(100 \mathrm{~Hz}, 1 \mathrm{~s}, 5$ times with $10 \mathrm{~s}$ interval). Five superimposed traces are shown for each condition. The $10 \mu \mathrm{m}$ bicuculline was included throughout. Calibration: $10 \mathrm{pA}$, $50 \mathrm{~ms}$. $\boldsymbol{B}$, Plot of evoked EPSC amplitude before and after HFS from a representative recording. In this example, both the number of stimuli that evoked successful EPSCs was increased and the increased amplitude of the EPSCs lasted for $\geq 30 \mathrm{~min}$. C1, The amplitude histogram distribution of control responses from a representative recording. C2, The amplitude histogram distribution after HFS. After HFS, there are significantly fewer failures, and the amplitude distribution reveals fewer small and more large amplitude events than under the control conditions (i.e., pre-HFS; minimal stimulus conditions). D, Pooled data of evoked EPSC amplitudes versus time in WT (average of 3 sequential responses, including failures; 9 pairs exhibited LTP after HFS). The post-HFS amplitudes are significantly increased in WT compared with the pre-HFS control. $\boldsymbol{E}$, Left, Summary data of EPSC potency for all experiments in WT mice. Evoked EPSC amplitudes (potency) of all positive (i.e., nonzero) EPSCs evoked with the minimal stimulus paradigm under control conditions versus after HFS. The overall effect of HFS was statistically significant ( $n=9$, $p=0.02$, paired sample Wilcoxon signed rank test ${ }^{*} p<0.05$ ). Right, Dot link plot of the changes in potency in each experiment, comparing control conditions to after HFS; 7 of 9 pairs showed an increased potency. $\boldsymbol{F}$, Left, Summary data of EPSC success probabilities for all experiments in WT mice. The overall effect of HFS was statistically significant $(n=9, p=0.004$, paired sample Wilcoxon signed rank test ${ }^{*} p<0.05$ ). Right, Dot link plot of the changes in success probability in each experiment comparing control conditions to after HFS; all 9 pairs showed an increased $P_{s}$.

because of their role in emotional memory (Jiang and Role, 2008). The plasticity of cortical glutamatergic inputs in WT and Nrg1 HET mice was compared by direct extracellular stimulation of the incoming cortical fiber tracts within the external capsule and concomitant recording of stimulus-evoked glutamatergic EPSCs (eEPSCs) in BLA pyramidal neurons before and after patterned stimulation of the cortical tract. Assay of eEPSCs elicited by a minimal stimulation protocol (i.e., stimulation intensity adjusted such that $\sim 50 \%$ of electrical stimuli elicited eEPSCs; see Materials and Methods) before and after a single high-frequency burst revealed robust and long-lasting potentiation of evoked glutamatergic transmission in WT mice (Fig. 3). The onset of potentiation typically began 1-3 min after HFS and persisted for at least $30 \mathrm{~min}$ (Fig. 3). Representative recordings and analysis of evoked transmission before and after a high-frequency burst are shown in Figure 3A-C. Analyses of these data revealed that plasticity is manifest in both increased amplitude and an increase in the fraction of successful single evoked responses (i.e., nonfailures) following the patterned stimulation (Fig. $3 \mathrm{~B}, \mathrm{C}$ ). A histogram of all of the evoked responses in this representative cell reveals that a single HFS burst elicits a right shift of the amplitude peaks and a reduced number of failures in response to subsequent $0.1 \mathrm{~Hz}$ stimulation (Fig. 3C1,C2). Most cortical-BLA synapses in the WT animals demonstrated this long-lasting potentiation of glutamatergic transmission (Fig. $3 D-F ; n=9$ ). HFS significantly increased the mean amplitude of eEPSCs (Fig. $3 D, E ; p=0.017$, paired sample Wilcoxon signed rank test, $n=9$ ). The observed success probability of eEPSCs was also significantly increased (Fig. 3F; $p=0.003$, paired sample Wilcoxon signed rank test, $n=$ 9). In sum, the HFS LTP was associated with increased potency of evoked EPSCs (Figure 3E; 7 of 9 tested) and increased success probability $\left(\mathrm{P}_{\mathrm{s}}\right.$, Fig. 3F; 9 of 9 tested).

In contrast to the robust and consistent effect of a single burst of HFS on long-lasting synaptic plasticity of cortical-BLA synapses in WT animals, we were able to evoke LTP at only a few cortical-BLA synapses in Nrgl HET mice using the same stimulation protocol ( $n=2$ of 9$)$. The overall mean amplitude was not changed by HFS (Fig. 4D). As shown in Figure $4 A$, some shortterm post-tetanic potentiation (PTP) was recorded after HFS of cortical BLA synapses in the Nrg1 HET, but sustained changes in transmission were not observed in most recorded cells (Fig. $4 A-$ $C$ ). Neither the potency (Fig. $4 E$; nonzero events, $p=0.42$, paired sample Wilcoxon signed rank test, $n=9$ ) nor the success rate of eEPSCs was significantly affected by HFS burst (Fig. 4F; $p=0.49$, paired sample Wilcoxon signed rank test, $n=9$ ). These observations are consistent with the fact that type III Nrg1 is essential to the induction of LTP at cortical-BLA glutamatergic synapses.

Type III Nrg1 is capable of back-signaling (Bao et al., 2003; Hancock et al., 2008; Zhong et al., 2008; Canetta et al., 2011). Because the LTP induction is impaired in the type III Nrg1 HET animals, we tested whether acute stimulation of back-signaling (stimulated by adding recombinant ErbB4 extracellular domain proteins, sErbB) rescued the plasticity at cortical-BLA synapses. Brain slices from Nrg1 HET animals were incubated with $10 \mathrm{nM}$ soluble ErbB4 for at least $1 \mathrm{~h}$. Evoked EPSCs (evoked at 25\% of maximum response) were measured before and after HFS. Under these conditions, HFS consistently induced potentiation at WT but not Nrg1 HET synapses (Fig. 4G; WT, $n=6$; Nrg1 HET, $n=$ $7 ; p=0.003$, MW test). Preincubation with soluble ErbB4 restored HFS induced potentiation in 3 of 9 tested Nrg1 HET neurons (Fig. $4 G$; Nrg1 HET, $n=7$; HET + sErBb, $n=3 ; p=0.04$, MW test). This rescue is most apparent when one considers the responses of individual cells (Fig. 4G, right); HFS induced potentiation in all WT neurons tested (6 of 6), 14\% of Nrg1 HET neurons (1 of 7), and 33\% of Nrg1 HET neurons from slices pretreated with soluble ErbB4 (3 of 9). Thus, type III Nrg1 backsignaling appears to contribute to HFS elicited LTP at these cortical-BLA glutamatergic synapses.

The observed lack of potentiation in Nrg1 HET animals could result from a fundamental impairment on the underlying mechanisms of potentiation or reflect a change in the threshold necessary to induce potentiation. To address this question, we increased the strength of stimuli used to evoke EPSCs to $20-25 \%$ of maximal response (Tsvetkov et al., 2004). The increase in stimulus did not change the extent of potentiation in WT (Fig. 5A; $10 \% \operatorname{MAX}, n=9 ; 25 \% \operatorname{MAX}, n=6 ; p=0.60$, MW test) or $\mathrm{Nrg} 1$ 

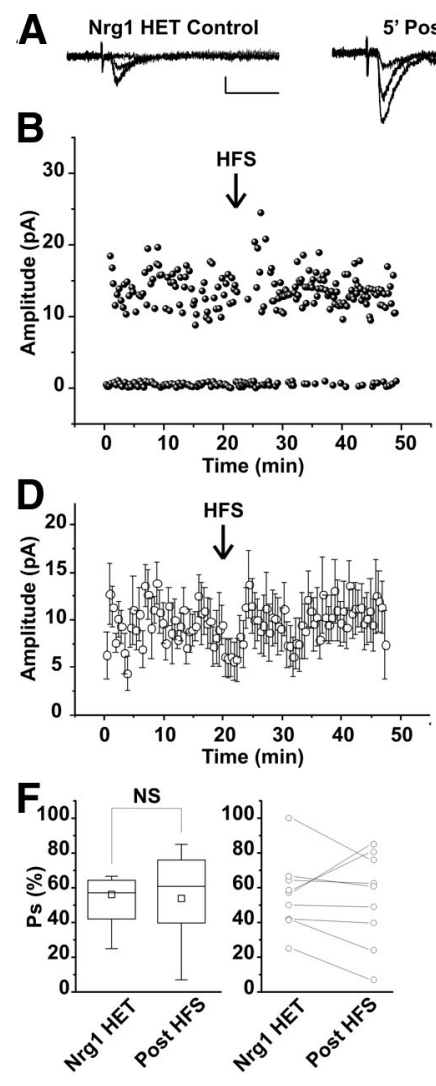

G
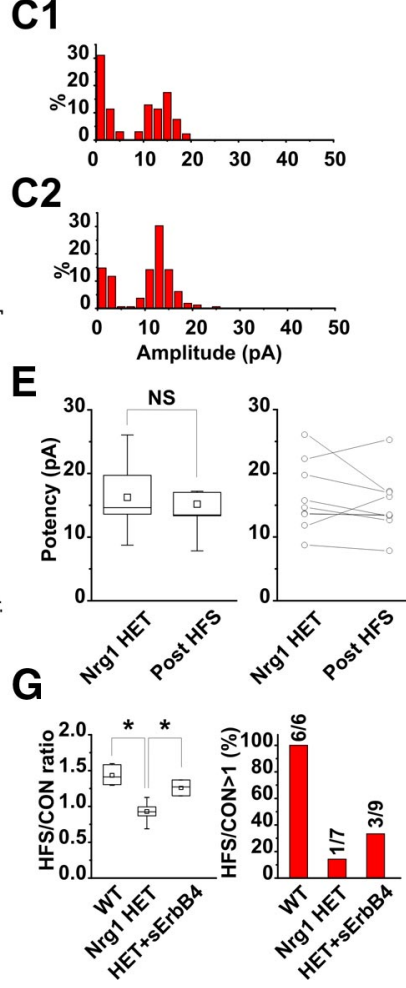

Figure 4. High-frequency burst stimulation failed to elicit LTP in cortical-BLA circuits in Nrg1 HET mice. $\boldsymbol{A}$, Three overlapping sample traces of evoked EPSC during control conditions (pre-HFS; minimal stimulation conditions as delineated in Fig. 3 legend), at 5 min and at $30 \mathrm{~min}$ after a high-frequency burst in a representative BLA pyramidal neuron from $\mathrm{Nrg} 1 \mathrm{HET}$ animals. HFS elicited only a brief PTP of transmission. During the brief PTP of $\sim 5 \mathrm{~min}$ after HFS, there is an increase of success rate and amplitude. There is typically no effect of HFS after the brief PTP in the Nrg1 HET animals. Calibration: 10 pA, 50 ms. B, Plot of evoked EPSC amplitude before and after HFS from a representative experiment in $\mathrm{Nrg} 1 \mathrm{HET}$ mice. C1, The amplitude histogram distribution of control conditions from a representative experiment in Nrg1 HET mice. C2, The amplitude histogram distribution after HFS from the same representative experiment in $\mathrm{Nrg} 1$ HET mice. D, Pooled data of evoked EPSC amplitudes versus time (average of 3 sequential responses, including failures; 7 of 9 pairs displayed no LTP after HFS in Nrg1 HET). The post-HFS amplitudes are not significantly increased. $\boldsymbol{E}$, Left, Summary data of EPSC potency for all experiments in Nrg1 HET mice. Evoked EPSC amplitudes (potency) of all positive (i.e., nonzero) EPSCS evoked with the minimal stimulus paradigm under control conditions versus after HFS in Nrg1 HET mice. The overall effect of HFS was not statistically significant $(n=9, p=0.43$, paired sample Wilcoxon signed rank test). NS, Not significant. Right, Dot link plot of the changes in amplitude in individual experiments comparing the amplitude of evoked responses under control conditions with post-HFS in Nrg1 HET mice. F, Left, Summary data of EPSC success probabilities for all experiments in Nrg1 HET mice. The overall effect of HFS was not statistically significant ( $n=9, p=0.50$, paired sample Wilcoxon signed rank test). NS, Not significant. Right, Dot link plot of the changes in success probability in each experiment in Nrg1 HET mice, comparing control conditions with post-HFS. NS, Not significant. G, Left, The extent of potentiation is represented as the ratio of post-HFS ( $>30 \mathrm{~min}$ ) to pre-HFS (control) amplitude for each experiment. Under the stimulation conditions used (pre-HFS: $20-25 \%$ of maximum response amplitude), there was a significant increase in EPSC amplitude after HFS in all WT mice tested (range, 1.35-1.65 HFS/CON ratio, 6 of 6), In contrast, the HFS/CON ratio was significantly decreased in Nrg1 HET animals (WT, $n=6 ;$ Nrg1 HET, $n=7 ; p=0.003$, MW test, ${ }^{*} p<0.05$ ). Treatment of slices from $\mathrm{Nrg} 1 \mathrm{HET}$ mice with soluble ErbB4 elicited significantly rescues the extent of the potentiation elicited by HFS ( $\mathrm{Nrg} 1 \mathrm{HET}, n=7$; Nrg1 HET treated with soluble extracellular domain of ErbB4, $n=3 ; p=0.04, \mathrm{MW}$ test, ${ }^{*} p<0.05$ ) in one-third of tested cells ( 3 of 9). Right, Bar plot of the percentage of total experiments in which the HFS/CON ratio $>1$ under different conditions: WT, 100\% (6 of 6); Nrg1 HET, 14.3\% (1 of 7); and Nrg1 HET treated with soluble extracellular domain of ErbB4, 33.3\% (3 of 9). Soluble ErbB significantly increased the percentage of neurons in which the HFS/CON ratio was increased compared with Nrg1 HET controls.
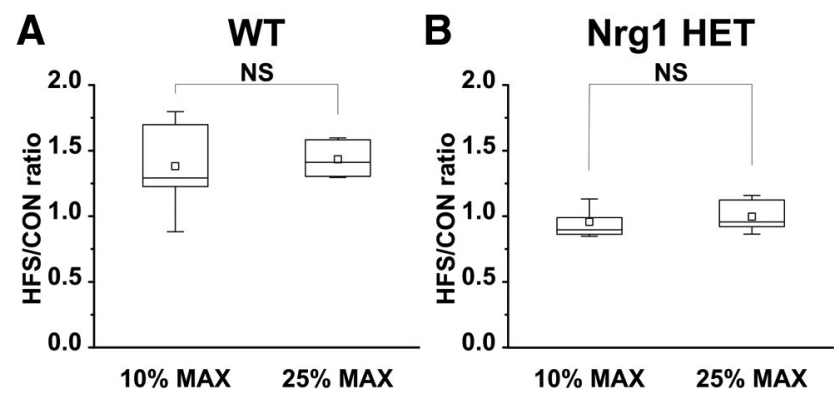

Figure 5. HFS-induced synaptic plasticity is independent of stimulus strength in both WT and Nrg1 HET mice. Comparison of WT $(\boldsymbol{A})$ and $\operatorname{Nrg} 1$ HET $(\boldsymbol{B})$ mice before and after HFS burst stiumulation when the baseline stimulation was adjusted to elicit $\sim 10 \%$ of the maximal response amplitude or $\sim 25 \%$ of the maximal response amplitude. The extent of potentiation (or lack thereof in the case of the $\mathrm{Nrg} 1 \mathrm{HET}$ ) was not significantly different for the two stimulation paradigms tested. $\boldsymbol{A}$, WT LTP was reliably elicited in both conditions. There is no significant difference in the potentiation ratio ( $10 \% \operatorname{MAX}, n=9 ; 25 \% \operatorname{MAX}, n=6 ; p=0.60$, MW test). NS, Not significant. $\boldsymbol{B}, \mathrm{Nrg} 1 \mathrm{HET}$. There is no significant difference in the potentiation ratio (10\% $\operatorname{MAX}, n=9 ; 25 \% \operatorname{MAX}, n=6 ; p=0.38$, MW test). NS, Not significant.

HET animals (Fig. 5B; 10\% MAX, $n=9 ; 25 \% \operatorname{MAX}, n=6 ; p=$ 0.38, MW test).

Type III Nrg1 is required for $\mathrm{nAChR}$-induced facilitation of glutamatergic transmission in BLA

We examined the expression of type III Nrg1 and $\alpha 7^{\star} \mathrm{nAChRs}$ in the cingulate cortex and the amygdala. Immunostaining with a type III Nrg1-specific antibody (Yang et al., 1998; Chen et al., 2010a, 2010b; Hancock et al., 2011) revealed expression in cortical pyramidal neurons (Fig. 6A). Costaining with $\alpha$-BgTx, which specifically binds to $\alpha 7^{\star}$ nAChRs in the CNS, demonstrated coexpression of $\alpha 7^{\star} \mathrm{nAChRs}$ in deep layer cortical neurons (Fig. $6 A)$. To determine whether cortical neurons that express $\alpha{ }^{\star}$ nAChRs project to the BLA, we injected the cingulate cortex with the anterograde tracer BDA (see Materials and Methods) and, after $5 \mathrm{~d}$, stained sections of the external capsule and the BLA with $\alpha$-BgTx. Figure $6 B$ shows labeling of $\mathrm{BDA}^{+}$cortical projections in both the EC and the BLA with $\alpha$-BgTx, confirming that cortical neurons that innervate BLA express $\alpha 7^{\star}$ nAChRs along their axons.

As type III Nrg1 has been implicated in the regulation of both the expression and targeting of nAChRs in other CNS and PNS neurons, we tested whether heterozygous deletion of type III Nrg1 altered nicotine-induced plasticity of glutamatergic transmission at synapses in the BLA (Jiang and Role, 2008). In our previous study, we have found that a brief exposure to nicotine ( $2 \mathrm{~min}, 500 \mathrm{nM}$ ) facilitated glutamatergic transmission to pyramidal neurons in the BLA of WT mice. In the presence of TTX $(1 \mu \mathrm{M})$ to monitor miniature synaptic currents, nicotine elicited a robust increase in the frequency of glutamatergic miniature EPSCs in $\sim 50 \%$ of neurons tested in WT mice $(n=17)$. The effects of nicotine were reflected in a rapid (within seconds) and significant increase in mEPSC frequency (Fig. 6C,E,I; WT control vs WT + nicotine, $p<0.001$, paired sample Wilcoxon signed rank test, $n=17$ ), without a detectable effect on mEPSC amplitude (Fig. $6 C, G, J$; WT control vs WT nicotine, $p=0.16$, paired sample Wilcoxon signed rank test). Interevent intervals of mEPSCs, but not of mEPSC amplitude, were significantly different in control versus nicotine conditions in the WT (Fig. 6E; $p=$ 0.003 , KS test, $n=17$; Fig. $6 G ; p=0.97$, KS test, $n=17$ ). Together, these data are consistent with the fact that a presynaptic mechanism contributes to the nicotine-induced facilitation of 


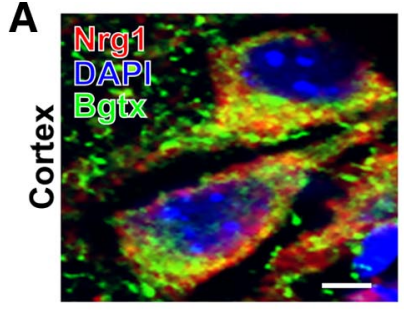

C

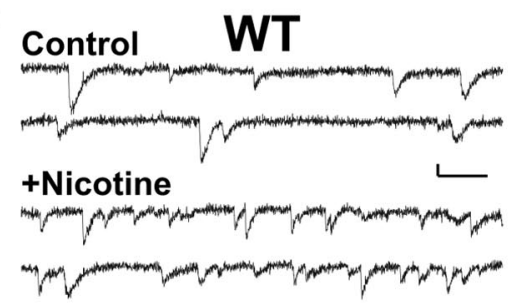

E

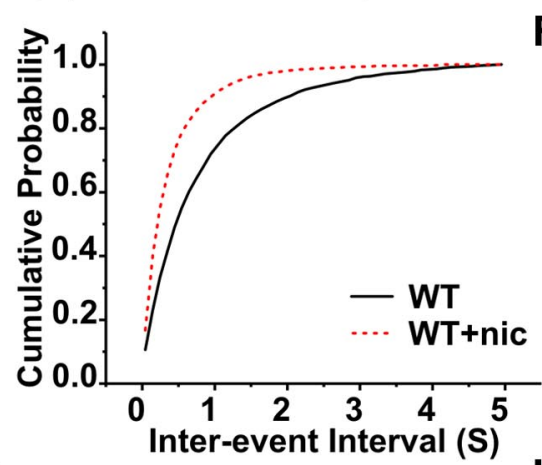

G
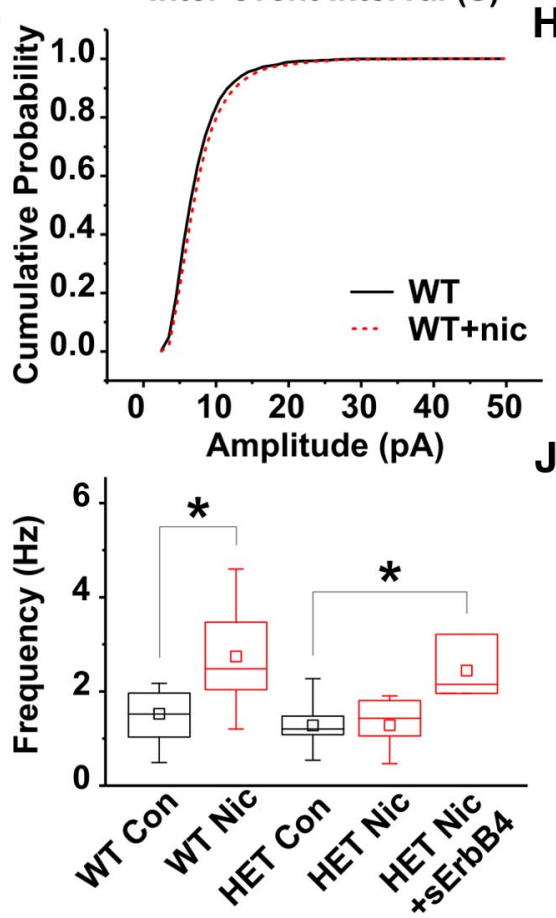

D
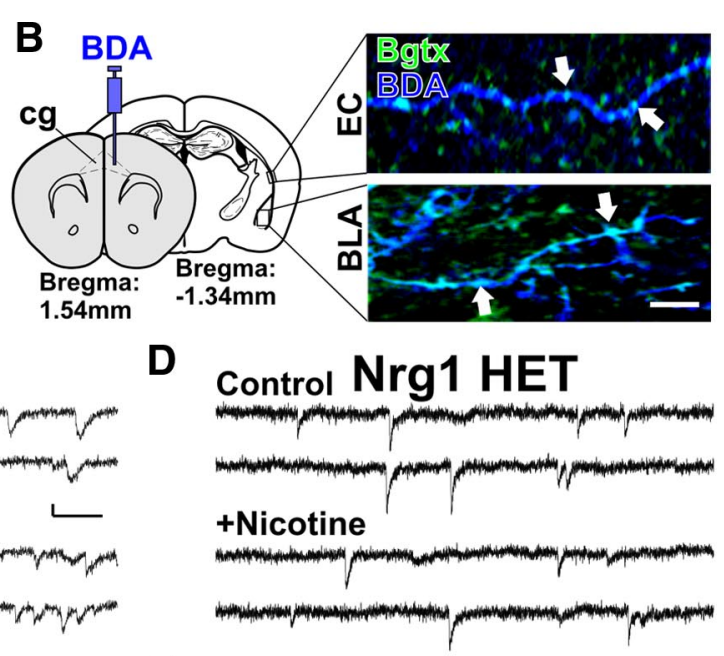

$F$

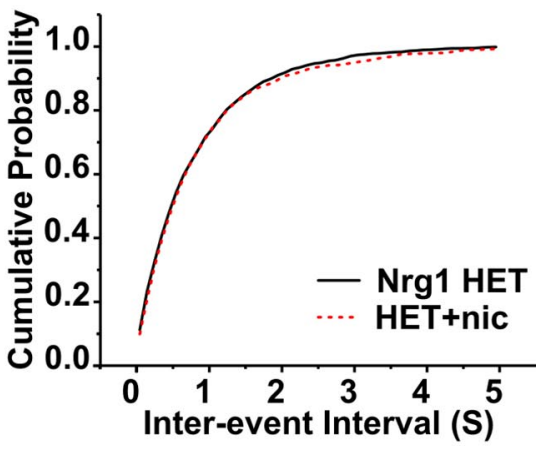

$\mathrm{H}$
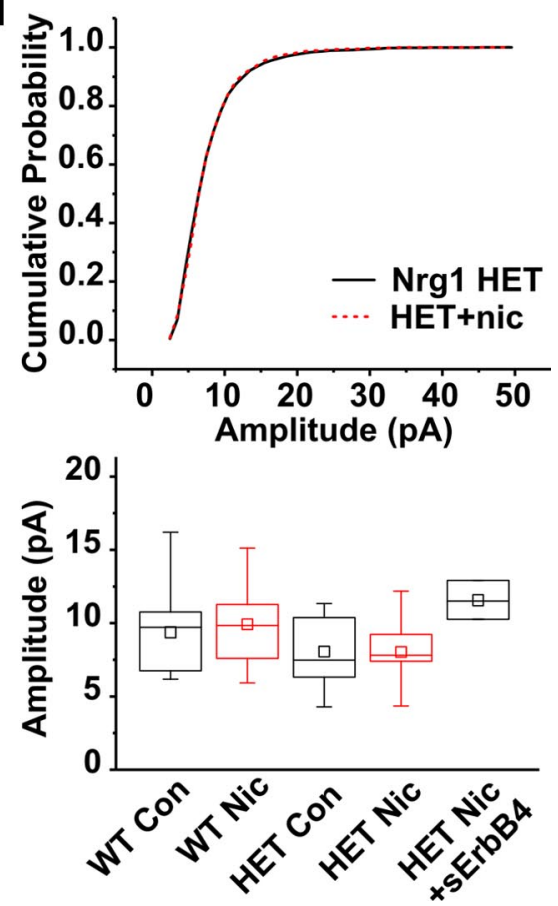

Figure 6. Nicotine elicits a robust facilitation of glutamatergic transmission in WT but not in Nrg1 HET mice. $\boldsymbol{A}$, Representative confocal fluorescence image from cingulate cortex layer 5 pyramidal neurons after staining with an antibody to type III Nrg 1 (red) and biotinylated $\alpha$-BgTx (green). Pyramidal neuron cell bodies in the cingulate cortex express both type III Nrg 1 and the BgTx target, consistent with their expression of $\alpha 7$-containing nicotinic acetylcholine receptors ( $\left.\alpha 7^{*} \mathrm{nAChR}\right)$. $\boldsymbol{B}$, Left, Schematic diagrams (adapted from Paxinos and Franklin, 2004), illustrating the injection of BDA into layer 5 of cingulate cortex and the location of micrographs of the labeled projections within the external capsule (EC; $\boldsymbol{B}$, right top) and labeled axons within the BLA ( $\boldsymbol{B}$, right bottom). Fluorescent images of cingulate cortical projections within the EC and BLA, where BDA fibers are labeled in dark blue and BgTx labeled in green, show multiple areas of overlap (in cyan; white arrowheads) consistent with $\alpha 7^{*} \mathrm{nAChRs}$ being localized along cingulate projections and at sites of termination within the BLA. Scale bars: $\boldsymbol{A}, 5 \mu \mathrm{m} ; \boldsymbol{B}$ (right), $5 \mu \mathrm{m}$. $\boldsymbol{C}$, Top, Sample traces of $\mathrm{mEPSC}$ recorded in a representative BLA neuron in acute slice from WT mice at a holding potential $\left(\mathrm{V}_{\mathrm{h}}\right)$ of $-60 \mathrm{mV}(1 \mu \mathrm{m}$ TTX glutamatergic transmission at corticalBLA synapses, as previously described.

To test whether nicotinic modulation of glutamatergic transmission was also observed in type III Nrg1 heterozygous animals, we first assessed mEPSCs in recordings from BLA pyramidal neurons in Nrg1 HET mice $(n=9)$. As delineated above, baseline glutamatergic synaptic input to BLA pyramidal neurons in Nrg1 HET animals was not significantly different from that observed in their WT littermates (Fig. $1 C)$. Neither the frequency nor the amplitude of glutamatergic mEPSCs in $\mathrm{Nrgl}$ HET animals was significantly different from those recorded in WT littermates (Fig. 1D,E), although the time constant or synaptic current decay is significantly faster in $\mathrm{Nrg} 1 \mathrm{HET}$ compared with WT littermate control.

In contrast to baseline synaptic activity, the nicotinic modulation of mEPSCs

$\leftarrow$

$10 \mu \mathrm{m}$ bicuculline, and $200 \mathrm{~nm}$ atropine). Calibration: 5 pA, 100 ms. Bottom, Sample traces of mEPS(s after brief application of nicotine ( $500 \mathrm{~nm}, 60 \mathrm{~s}$ ). Calibration: 5 pA, 100 ms. D, Sample traces of $\mathrm{mEPS}(\mathrm{s}$ recorded in a representative BLA neuron in acute slice from $\mathrm{Nrg} 1 \mathrm{HET}$ mice. All conditions and scales as in A. $E$, Cumulative distribution of the $m E P S C$ interevent intervals in WT mice $(n=17)$ demonstrating the facilitatory effect of acute nicotine on spontaneous glutamatergic synaptic transmission. The cumulative plot is significantly left shifted from the control distribution after brief exposure to nicotine. Such a left shift is indicative of an increased mEPSC frequency and consistent with previous reports that nicotine causes an increase in the probability of glutamate release at synapses on BLA pyramidal neurons (solid line, WT control; dotted line, WT + nicotine) (see Jiang and Role, 2008). WT control versus WT + nicotine are significantly different $(n=17, p=0.003$, KS test). $\boldsymbol{F}$, Cumulative distribution of the mEPSC interevent intervals recorded in $\operatorname{Nrg} 1$ HET mice $(n=9)$. The cumulative distribution of mEPSC interevent interval times was not significantly different between $\mathrm{Nrg} 1 \mathrm{HET}$ and $\mathrm{Nrg} 1 \mathrm{HET}+$ nicotine, indicating similar mEPSC frequency (solid line, HET control; dotted line, HET + nicotine; $n=9, p=0.40, \mathrm{KS}$ test). $\boldsymbol{G}, \boldsymbol{H}$, Cumulative distribution of mEPSC amplitude in WT and Nrg1 HET mice: acute nicotine exposure was without effect on the amplitude of mEPSCs in both WT and Nrg1 HET mice (G, solid line, WT control; dotted line, WT + nicotine; $n=17, p=$ 0.97, KS test; $\boldsymbol{H}$, solid line, HET control; dotted line, HET + nicotine; $n=9, p=0.07$, KS test). $I$, Population data of mEPSC frequency WT versus Nrg1 HET. Nicotine significantly increased the mEPSC frequency in WT but not Nrg 1 HET mice (WT, $n=17, p<0.0001 ;$ HET,$n=9 ; p=1$; paired sample Wilcoxon signed rank test, ${ }^{*} p<0.05$ ). Treatment of slices from $\mathrm{Nrg} 1 \mathrm{HET}$ mice with soluble ErbB4 elicited significant rescue of the extent of the facilitation induced by acute nicotine in 3 of 11 cells (HET Con, $n=9$; HET Nic + sErbB, $n=3 ; p=$ 0.02 , MW test). ${ }^{*} p<0.05$. J, Population data of mEPSC amplitudes. Nicotine had no effect on $m E P S C$ amplitude in either WT or Nrg1 HET BLA pyramidal neurons (WT, $n=17, p=$ 0.16 ; HET, $n=9, p=0.65$; paired sample Wilcoxon signed rank test). Treatment with soluble ErbB4 did not significantly affect the mEPSC amplitude (HET Con, $n=9$; HET Nic + sErbB, $n=3 ; p=0.06$, MW test). 
recorded in BLA pyramidal neurons in Nrg1 HET mice was significantly different from WT. Unlike their WT littermates, treatment with nicotine was without effect on mEPSC frequency or amplitude at $100 \%$ of synapses tested in the BLA from $\mathrm{Nrg1}$ HET animals (Fig. $6 D, F, H$ ). The cumulative distribution plots of mEPSC interevent intervals (Fig. $6 F ; p=0.4$, KS test, $n=9$ ) and amplitude (Fig. $6 H ; p=0.07$, KS test, $n=9$ ) showed no significant difference before and after treatment with nicotine. Box plot analysis of the pooled data reveal the same lack of effect of nicotine on mEPSC frequency or amplitude in the Nrg1 HET (Fig. 6I; HET control vs HET nicotine, $p=1$, paired sample Wilcoxon signed rank test, $n=9$; Fig. 6 J; HET control and HET nicotine, $p=0.65$, paired sample Wilcoxon signed rank test, $n=9$ ). Our findings demonstrate that the heterozygous deletion of type III $\mathrm{Nrg} 1$ eliminates nicotine-mediated modulation of mEPSCs in the BLA.

To examine whether acute stimulation of type III Nrg1 backsignaling could rescue nicotinic facilitation in the Nrg1 HET animals, amygdala slices from $\mathrm{Nrg} 1 \mathrm{HET}$ animals were treated with soluble ErbB4-ECD. ErbB4-ECD treatment rescued nicotinic facilitation of mEPSC frequency in 3 of 11 cells (Fig. 6I; HET Con, $n=9$; HET Nic + sErbB4, $n=3 ; p=0.02$, MW test), without affecting mEPSC amplitude (Fig. 6J; HET Con, HET Nic + sErbB4, $p=0.06$, MW test). These results are consistent with a key role of type III $\mathrm{Nrg} 1$ in regulating levels of a subset of presynaptic nAChRs, presumably the $\alpha 7^{\star}$ nAChRs (Zhong et al., 2008).

\section{Nicotine facilitates TBS elicited LTP of cortical-BLA synaptic transmission in WT mice}

Prior studies of cortical-BLA circuits demonstrated that TBS of cortical inputs typically elicits short-term enhancement of excitatory synaptic plasticity in BLA pyramidal neurons (Jiang and Role, 2008). However, when the TBS stimulation of cortical input is combined with a single exposure to nicotine, there is a significant decrease of the threshold for activation of LTP (Fig. 7A1-A3) (Jiang and Role, 2008). Figure 7 summarizes findings from examination of WT littermates of Nrg1 HET mice, documenting the short-term facilitation elicited by TBS of cortical inputs to the BLA (Fig. 7A3; control and TBS, $p<0.001$, paired sample Wilcoxon signed rank test, $n=16$ ). Likewise, concurrent activation of cortical inputs with TBS and application of a single dose of nicotine (500 nM) typically elicited LTP, with near-doubling of the eEPSC amplitudes for 30-60 min in WT mice and in WT littermates of $\mathrm{Nrg} 1 \mathrm{HETs}$ (Jiang and Role, 2008) (Fig. 7A3; TBS + Nic, $p<0.001 ; 30$ min after $\mathrm{T}+\mathrm{N}, p<0.001$, paired sample Wilcoxon signed rank test, $n=16$ ). These findings demonstrate that cholinergic signaling at WT cortical-BLA glutamatergic synapses can play a critical role in the induction and/or maintenance of LTP.

\section{Nicotine does not induce LTP after TBS in type III Nrg 1 heterozygous mice}

We next tested whether the levels of expression of type III Nrg1 influenced the cholinergic modulation of TBS-induced synaptic plasticity in cortical-BLA circuits. To test this, we examined excitatory synaptic transmission at cortical-BLA synapses from Nrg1 HET mice before and after TBS and in the presence or absence of concomitantly applied nicotine (500 nM). As in the WT littermates, most pyramidal neurons responded to TBS with a short-term change in the amplitude of eEPSCs in Nrg1 HET animals (Fig. 7B1-B3; control and TBS, $p=0.04$, paired sample Wilcoxon signed rank test, $n=8$ ). However, in contrast to our
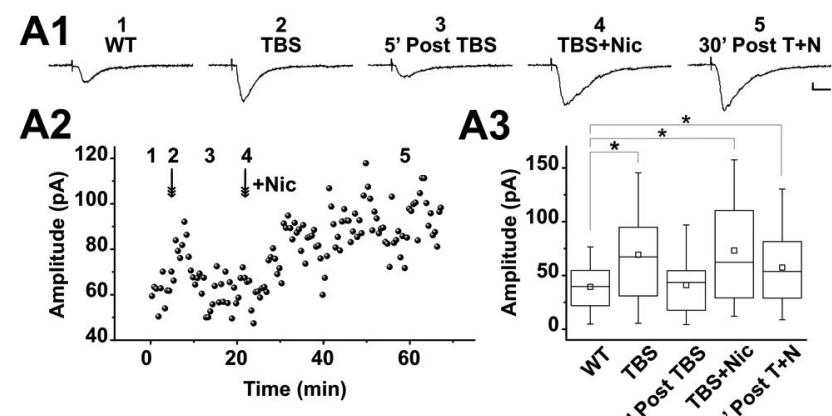
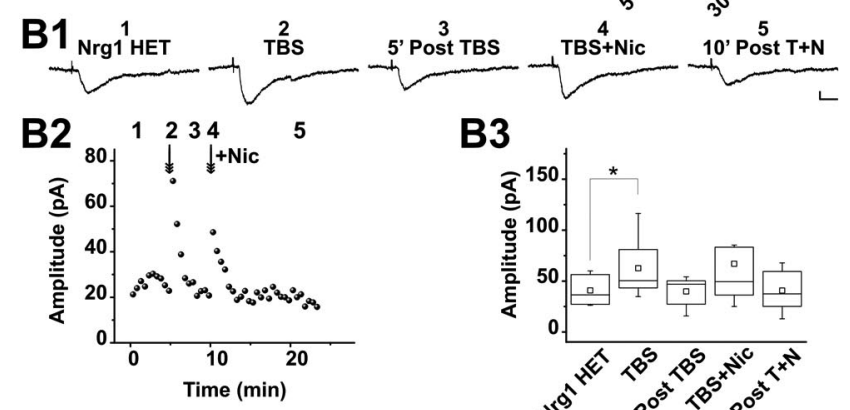

\section{B3}

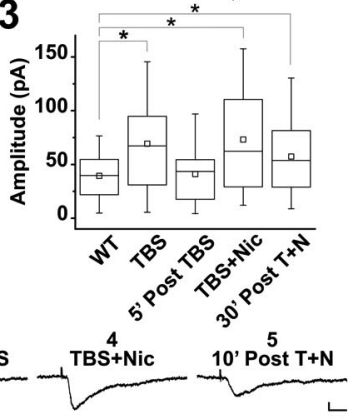

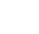
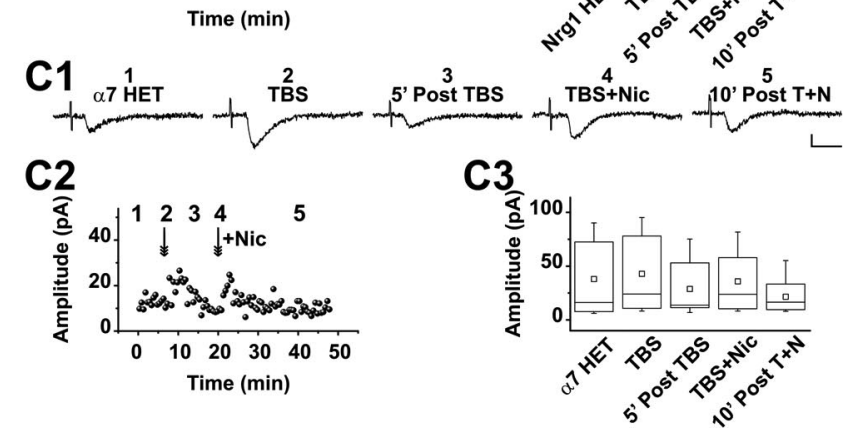

Figure 7. Nicotine lowers the threshold for TBS induced LTP of cortical-BLA synapses in WT mice, but not in mice heterozygous for either $\mathrm{Nrg} 1$ or the $\alpha 7 \mathrm{nAChR}$ subunit. A1, Representative evoked EPSC responses to $0.1 \mathrm{~Hz}$ stimulation of a WT neuron during control conditions (1, control), immediately after TBS (2,TBS), 5 min after TBS (3, 5 min after TBS), immediately after concurrent administration of nicotine and TBS (4, TBS + Nic), and 30 min after TBS + nicotine $(5,30$ min after $T+N)$. Sample records shown were taken at the time points as indicated in $\boldsymbol{B}$ (from left to right). Calibration: 20 pA, 25 ms. A2, Plot of evoked EPSC amplitudes recording from representative BLA pyramidal neuron with $0.1 \mathrm{~Hz}$ stimulation of cortical input (average of 3 sequential responses). Arrows indicate the time of delivery of TBS alone (first arrow) and the time of delivery of TBS + nicotine (second arrow; $+\mathrm{Nic}$ ). The traces are labeled to indicate the 1-5 corresponding time and conditions of the recording as shown in $\mathbf{A}$. A3, The averaged evoked EPSC amplitude for all WT neurons recorded $(n=16)$. TBS itself elicits brief potentiation (TBS, $p<0.0001$, paired sample Wilcoxon signed rank test). After $5 \mathrm{~min}$, the amplitudes drop back to control level ( 5 min after TBS, $p=0.23$ ). Exposure to nicotine converts this brief potentiation to sustained potentiation induced by TBS (TBS + Nic, $p<0.0001 ; 30$ min after $\mathrm{T}+\mathrm{N}, p<0.0001$, paired sample Wilcoxon signed rank test). ${ }^{*} p<0.05$. B1, Representative evoked EPSC traces from $\mathrm{Nrg} 1 \mathrm{HET}$ examined with the same protocol as in $\boldsymbol{A}$. Calibration: $10 \mathrm{pA}$, 25 ms. B2, Plot of evoked EPSC amplitudes versus time in Nrg1 HET (average of 3 sequential responses). TBS alone (first arrow) elicits brief potentiation as in WT. In contrast, TBS + nicotine (second arrow + Nic) is without effect in Nrg1 HET. B3, The averaged evoked EPSC amplitude for all Nrg1 HET neurons recorded ( $n=8$ ). TBS itself elicits a brief potentiation (TBS, $p=0.04$; 5 min after TBS, $p=0.84$; paired sample Wilcoxon signed rank test). Nicotine is without effect when paired with TBS in Nrg1 HET (TBS + Nic, $p=0.15 ; 30$ min after T $+N, p=0.64$, paired sample Wilcoxon signed rank test). ${ }^{*} p<0.05$. (1, Representative evoked EPSC responses from $\alpha 7$ HET using the same experimental protocol as in $\boldsymbol{A}, \boldsymbol{B}$. Calibration: $10 \mathrm{pA}, 20 \mathrm{~ms}$. C2, Plot of EPSC amplitudes recorded before and after TBS \pm nicotine in an $\alpha 7$ HET (average of 3 sequential responses). Nicotine is without effect on EPSCs with or without prior TBS in the $\alpha 7$ HET mice. C3, The averaged evoked EPSC amplitudes for all the neurons recorded in the $\alpha$ 7 HET ( $n=7)$. TBS does not elicit significant potentiation whether or not paired with nicotine application (TBS, $p=0.38,5$ min after TBS, $p=0.22 ; \mathrm{TBS}+\mathrm{Nic}, p=0.81 ; 30$ min after $\mathrm{T}+\mathrm{N}, p=0.23$, paired sample Wilcoxon signed rank test). 

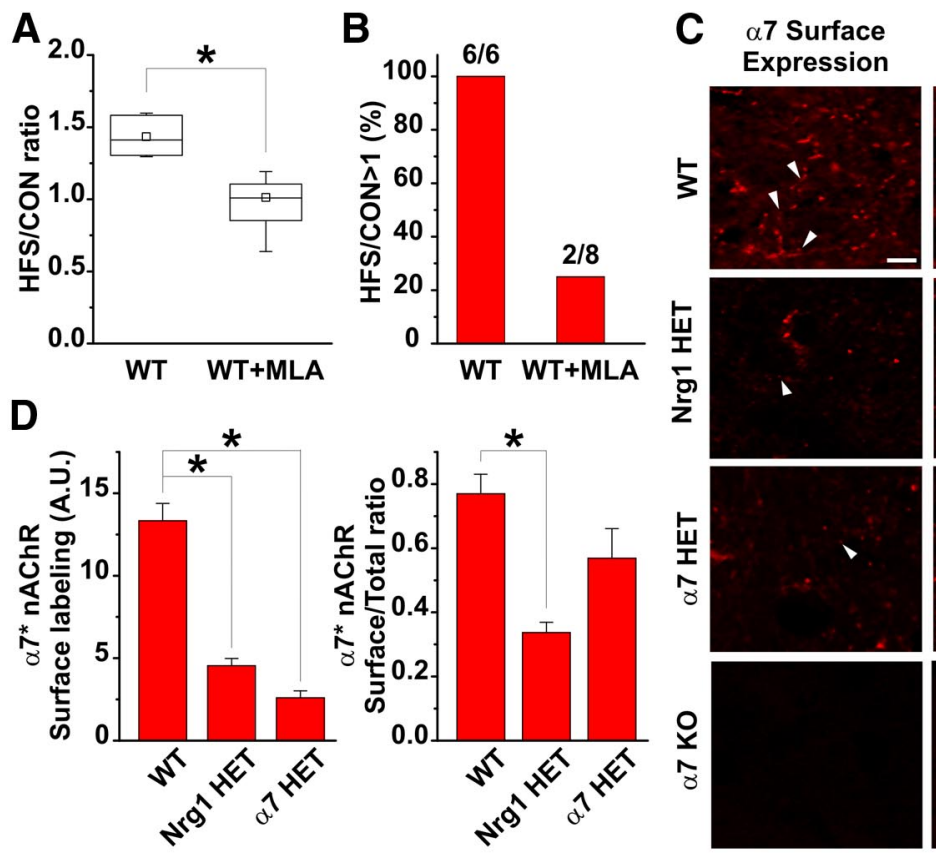

Figure 8. Role of $\alpha 7^{*} n A C h R s$ in type III Nrg1-dependent cortical-BLA synaptic plasticity. $\boldsymbol{A}, \boldsymbol{B}$, An $\alpha 7^{*}$ nAChR selective antagonist, MLA, administered at $10 \mathrm{~nm}$, significantly attenuated the extent of $L T P$ induction in WT animals. The effect of MLA was tested by comparison of the post-HFS to pre-HFS (control) amplitude ratios $(\boldsymbol{A})$ and by the number of experiments in which the $\mathrm{HFS} / \mathrm{CON}$ ratio was $>1(\boldsymbol{B})$. A total of $10 \mathrm{~nm}$ MLA significantly reduced HFSLTP (WT, $n=6 ; \mathrm{WT}+\mathrm{MLA}, n=8 ; p=0.01$, MW test). C, Confocal images of $\alpha$-BgTx (red) to detect surface and total $\alpha 7^{*}$ nAChR expression in the BLA of WT, Nrg 1 HET, $\alpha 7$ HET, and $\alpha 7$ K0 mice. Scale bar, $5 \mu \mathrm{m}$. Arrowheads indicate $\alpha 7^{*} \mathrm{nAChR}$ on fibers. ${ }^{*} \alpha 7^{*} \mathrm{nAChR}{ }^{+}$principal pyramidal-like cells within the BLA. $D$, Semiquantitative measurements of $\alpha 7$ surface expression (left) in the BLA of WT, Nrg1 HET, and $\alpha 7$ HET mice where the data were plotted as a ratio of the pixel volume of the surface expression over the pixel volume of total expression (right). Error bars represent SEM. ${ }^{*} p<0.001$ (left panel of surface labeling). ${ }^{*} p<0.01$ (right panel of surface/total ratio) by ANOVA followed by Bonferroni's post hoc test ( $n=3$ animals per genotype; 2 image fields per animal were analyzed and normalized to $\alpha 7 \mathrm{KO}$ background levels).

findings in WT mice, pairing of TBS with nicotine had no significant additional effect on either the extent or the duration of enhanced glutamatergic transmission in Nrgl HET mice (Fig. $7 B 1-B 3$; TBS + Nic, $p=0.15 ; 10$ min after $\mathrm{T}+\mathrm{N}, p=0.64$, paired sample Wilcoxon signed rank test, $n=8$ ). Figure $7 B 1, B 2$ shows representative tracings and time course of the effects of TBS + / - nicotine; Figure $7 B 3$ contains the population data $(n=$ 8 ). These data demonstrate that modulation of TBS-induced synaptic plasticity by $\mathrm{nAChR}$ activation is deficient in type III Nrg1 heterozygous animals.

$\alpha 7$ HET mice phenocopy the type III Nrg1 HET mice with respect to the effects of TBS and its modulation by nicotine Several prior studies demonstrate a prominent role for Nrg1 signaling in the regulation of expression and targeting of neuronal nAChRs (Hancock et al., 2008; Zhong et al., 2008). In particular, type III Nrg1 back-signaling has been shown to be linked to the regulation of targeted insertion of $\alpha 7$-containing nAChRs ( $\alpha 7^{\star}$ nAChRs) along axons (Hancock et al., 2008). To test whether the loss of nAChR modulation of synaptic transmission in $\mathrm{Nrg} 1$ HETs was the result, at least in part, of decreased levels of $\alpha 7$, we repeated a subset of the plasticity studies in mice heterozygous for $\alpha 7$ deletion ( $\alpha 7 \mathrm{HET}$ ). In particular, as summarized in Figure $7 C$, TBS of glutamatergic inputs to BLA in $\alpha 7$ HETs failed to elicit long-lasting synaptic potentiation whether or not the TBS stimulation was coupled with nicotine application (Fig. 7C3; TBS, $p=$ 0.38 ; TBS + Nic, $p=0.81 ; 10$ min after $\mathrm{T}+\mathrm{N}, p=0.22$, paired sample Wilcoxon signed rank test, $n=7$ experiments, 4 mice).
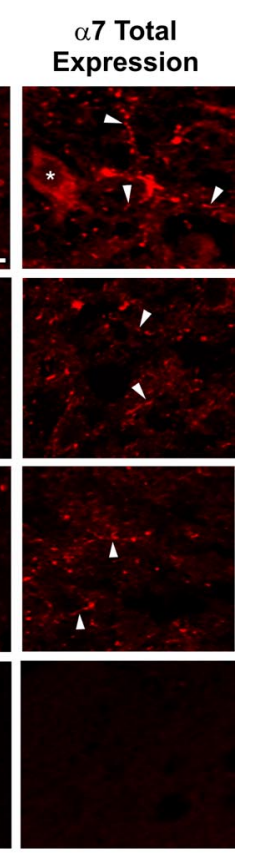

Only a subset of pyramidal neurons $(n=1$ of 7 neurons) showed even a brief increase in the amplitude of eEPSCs after TBS or combined TBS and nicotine (Fig. 7C1,C2). These data suggest that the activation of $\alpha 7$-containing nicotinic receptors is required for the induction of LTP of glutamatergic transmission at synapses onto pyramidal neurons in the BLA.

\section{Block of $\alpha 7$ receptor attenuates LTP induction in WT animals}

To determine whether $\alpha 7^{\star} \mathrm{nAChRs}$ contribute to HFS induced potentiation of cortical-BLA synapses in WT mice, we repeated the HFS analysis in WT slices in the absence and presence of the $\alpha 7$-selective nAChR antagonist, methyllycaconitine (MLA). Consistent with the results presented above, HFS elicited reliable LTP in WT neurons (100\%, 6 of 6 cells). In contrast, $10 \mathrm{~nm}$ of MLA attenuated LTP induction in WT animals. In the presence of MLA, HFS elicited LTP in only 25\% (2 of 8 ) cells (Fig. $8 A, B$; WT, $n=6$; WT +10 MLA, $n=8 ; p=0.01$, MW test). These findings support a crucial role for $\alpha 7^{\star} \mathrm{nAChRs}$ in mediating LTP at significant subset of cortical-BLA synapses.

As noted above, we have previously demonstrated that presynaptic targeting of $\alpha 7^{\star} \mathrm{nAChRs}$ is regulated by type III Nrg1 back-signaling (Hancock et al., 2008; Zhong et al., 2008), that cortical pyramidal neurons that innervate the BLA express both type III $\mathrm{Nrg} 1$ and $\alpha 7^{\star} \mathrm{nAChRs}$ (Fig. $6 A$ ), and that acute stimulation of type III Nrg1 back-signaling rescues HFS induced LTP at a subset of synapses in type III Nrg1 HET animals (Fig. 4G). These findings raise the possibility that one critical target of Nrgl signaling that is affected in the Nrg1 HET animals is presynaptic $\alpha 7^{\star} n A C h R s$. To examine this in more detail, we stained sections of BLA with $\alpha$-BgTx both before (to label surface receptors) and after permeabilization (for labeling of surface and intracellular $\alpha 7^{\star}$ nAChRs; Fig. $8 C$ ). Both surface and total $\alpha$-BgTx-stained fibers were visible in WT BLA. No staining of slices from $\alpha 7$ knock-outs animals was seen under either condition. Surface $\alpha 7^{\star} \mathrm{nAChR}$ and the surface/total ratio of $\alpha 7^{\star} \mathrm{nAChR}$ were reduced in type III Nrg1 HET (by $\sim 70 \%$ and 60\%, respectively; total $\alpha 7^{\star}$ nAChR levels were decreased slightly in the $\mathrm{Nrg} 1$ HET). Both surface and total levels of $\alpha 7^{\star} \mathrm{nAChR}$ were reduced in the $\alpha 7$ HET BLA (by $\sim 80 \%$ and $70 \%$, respectively) so that the surface/total ratio of $\alpha$-BgTx was not significantly changed (Fig. $8 C, D$; WT, $n=6$; Nrg1 HET, $n=6$; $p<0.01 ; \alpha 7 \mathrm{HET}, n=3 ; p=$ $0.11)$. These results are consistent with the conclusions that targeting of $\alpha 7^{\star} \mathrm{nAChRs}$ to presynaptic sites is regulated by type III Nrg1 and that the presynaptic $\alpha 7^{\star}$ nAChRs are a crucial contributor to plasticity of cortical-BLA synapses.

\section{Discussion}

The major findings of this study are that mice with only one functional allele of the type III Nrg1 gene lack multiple aspects of excitatory synaptic plasticity in cortical-BLA circuits. Specifically, in type III Nrgl heterozygotes, cortical-BLA synapses failed 
to potentiate in response to either HFS (Fig. 3 vs Fig. 4) or TBS in combination with brief exposure to low concentrations of nicotine, conditions that reliably induce sustained potentiation of synaptic transmission in WT littermates (Fig. 7) (Jiang and Role, 2008). This latter result was phenocopied in $\alpha 7^{\star} \mathrm{nAChR}$ heterozygotes (Fig. 7). In addition to these dramatic changes in plasticity, more subtle differences between type III Nrg1 heterozygotes and WT mice were seen in terms of baseline synaptic transmission. Most notably, we found a decrease in NMDA/AMPA ratio, largely driven by increased AMPA receptor-mediated synaptic current amplitude (Fig. 2), an accelerated rate of decay of mEPSCs (Fig. 1), and a failure of nicotine to increase mEPSC frequency (Fig. 6).

These studies add the cortical-BLA synapse to a growing roster of circuits in which the strength of Nrg1/ErbB4 signaling plays a critical role in modulating activity-dependent plasticity (Bao et al., 2003, 2004; Li et al., 2007; Buonanno et al., 2008; Neddens et al., 2009; Chen et al., 2010b; Shamir et al., 2012). However, the current study identifies a unique role for type III Nrg1 in circuit modulation. In general, acute stimulation of ErbB signaling in the hippocampus inhibits or reverses LTP, a result that is supported by genetic deletion of ErbB4. Genetic disruption of Nrg1 (affecting all isoforms) creates a more complex picture: Bjarnadottir et al. (2007) reported deficits in TBS, but not tetanusinduced hippocampal LTP in Nrgl heterozygous animals, and increased paired pulse facilitation, differences that were reversed by low, but not high, concentrations of exogenous Nrgl peptide. In the BLA, we examined the ability of either a HFS or a $\theta$ burst stimulus to elicit LTP. The HFS paradigm probes the effects of a single high-frequency burst of activity on subsequent synaptic responses; $\theta$ burst patterned stimulation and its modulation by nicotine assess more fine-tuned deficits in synaptic plasticity and the possible role of changes in presynaptic modulation. We found that type III Nrg1 heterozygotes fail to develop LTP in response to either tetanus or a $\theta$ burst + nicotine protocol, raising the likelihood that reducing type III Nrgl affects the modulation of these circuits by a different mechanism than proposed for the effects of Nrg1-ErbB4 signaling in cortex and hippocampus (Pitcher et al., 2008; Chen et al., 2010b).

Our results clearly demonstrate that normal levels of type III Nrg1 are required for plasticity at cortical-amygdala synapses. Acute stimulation of type III Nrg1 back-signaling restored plasticity at a subset of these synapses in type III Nrg1 HET BLA (Fig. 4G). Based on these rescue experiments, we conclude that ongoing type III Nrg1 signaling in the BLA is essential for potentiation of cortical-BLA synapses. It is important to note that type III Nrg1 is expressed both in the cortical neurons that project to the BLA (Figs. $1 A$ and Fig. $6 A$ ), and in a subset of BLA neurons (our unpublished observations). As a result, lack of type III Nrg1 back-signaling in either presynaptic inputs, postsynaptic BLA neurons, or both, could contribute to the changes in cortical-BLA synaptic transmission observed in the type III Nrg1 HETs.

Cortical amygdala synapses in $\alpha 7^{\star} \mathrm{nAChR}$ heterozygotes fail to potentiate in response to combined $\theta$ burst and nicotine stimulation (Fig. 7); the $\alpha 7^{\star}$ nAChR selective antagonist MLA blocks potentiation at these synapses (Fig. 8), and there is a substantial reduction in surface $\alpha 7^{\star} \mathrm{nAChRs}$ in the BLA of type III Nrg1 heterozygotes (Fig. 8). Type III Nrg1 regulates the level of expression (Yang et al., 1998; Mathew et al., 2007) and the presynaptic targeting (Hancock et al., 2008; Zhong et al., 2008) of $\alpha 7^{\star} \mathrm{nAChRs}$. As such, decreased expression and/or altered loss of sufficient functional presynaptic $\alpha 7^{\star} \mathrm{nAChRs}$ might be one factor accounting for the failure of nicotine to either increase spontaneous activity or to effectively synergize with patterned stimulation in the BLA. Given the similarities in nicotine responsiveness of cortical-BLA synapses and the surface $\alpha 7^{\star} \mathrm{nAChR}$ levels between the type III Nrg1 HETs and the $\alpha 7 n A C h R$ HETs (Figs. 7, 8), as well as the requirement for $\alpha 7^{\star} \mathrm{nAChR}$ for cortical-BLA plasticity (Fig. $8 A, B$ ), we propose that type III Nrg1 back-signaling is required for presynaptic targeting of $\alpha 7^{\star} \mathrm{AChRs}$ to cortical-BLA synapses.

Acute stimulation of type III Nrg1 back-signaling in BLA slices partially rescued cortical-BLA synaptic plasticity and nicotine responsiveness at a subset of synapses tested. At present, we do not know why the rest of the synapses tested did not detectably respond to soluble ErbB4 treatment. One possibility is that type III Nrg1 back-signaling from distal projections back to the cortical cell soma (which are not present in the acute slice preparation) is also required at these synapses (Bao et al., 2003, 2004). Maintenance of WT levels of presynaptic $\alpha 7^{\star} \mathrm{nAChRs}$ has been shown to involve multiple mechanisms of downstream type III Nrg1 back-signaling; back-signaling through type III Nrg1 can elicit a local recruitment of preexisting internal $\alpha 7^{\star} \mathrm{nAChRs}$ to the cell surface and/or a protein synthesis-dependent targeting of $\alpha^{\star}$ nAChRs to axons (Hancock et al., 2008; Zhong et al., 2008). Failure to rescue plasticity and nicotine responses at some cortical-BLA synapses might indicate a requirement for the latter mechanism. A second possibility is that there is insufficient presynaptic type III Nrg1 at the cortical-BLA synapses to support a full response to acute soluble ErbB4 stimulation (as in Canetta et al., 2011). The amount of type III Nrg1 protein within layer 5 cortical neurons appears to be reduced substantially in the Nrg1 HET animals (Fig. 1A), consistent with a reduced efficacy of back-signaling in these cortical projections. A third possibility is that lack of type III Nrg1-ErbB4 forward signaling also contributes to the deficit in corticalBLA synaptic plasticity in the type III Nrgl heterozygotes. ErbB4 is expressed on a small population of GABAergic interneurons within the BLA (Shamir et al., 2012), a population of neurons that plays an important role in the overall activity within the BLA (Ehrlich et al., 2009). Preliminary analyses of cortical-amygdala synaptic plasticity in ErbB4 knock-out animals reveal a phenotype intermediate between WT and the type III Nrg1 heterozygotes.

Although much of our focus in this study is on the presynaptic role of type III $\mathrm{Nrg} 1$ and $\alpha 7^{\star} \mathrm{nAChRs}$ at cortical-BLA synapses, we also observed alterations of several characteristic of the postsynaptic BLA neurons, including altered mEPSC decay kinetics and altered NMDA/AMPA ratios. At present, we do not know whether these postsynaptic changes result from altered type III Nrg1 back-signaling within the BLA pyramidal neurons (a significant fraction of which express type III Nrg1), or are secondary to either alterations in cortical inputs or alterations in ErbB4expressing GABAergic interneurons.

The possible functional interaction between type III Nrg1 and $\alpha 7^{\star} \mathrm{nAChR}$ is especially intriguing given the significant evidence from genetic studies implicating both NRG1 and CHRNA7 as schizophrenia susceptibility genes (Freedman et al., 2001; Stefansson et al., 2002; Harrison and Weinberger, 2005; Leonard and Freedman, 2006). Activation of $\alpha 7^{\star}$ nAChRs by acetylcholine might be an important regulator of cortical-BLA and other cortical-limbic circuits that are effected in schizophrenia patients. 


\section{References}

Abe Y, Namba H, Kato T, Iwakura Y, Nawa H (2011) Neuregulin-1 signals from the periphery regulate AMPA receptor sensitivity and expression in GABAergic interneurons in developing neocortex. J Neurosci 31: 5699-5709. CrossRef Medline

Arlotta P, Molyneaux BJ, Chen J, Inoue J, Kominami R, Macklis JD (2005) Neuronal subtype-specific genes that control corticospinal motor neuron development in vivo. Neuron 45:207-221. CrossRef Medline

Bae JS, Pasaje CF, Park BL, Cheong HS, Kim JH, Kim JY, Shin JG, Park CS, Kim BJ, Lee CS, Lee M, Choi WH, Shin TM, Hwang J, Shin HD, Woo SI (2012) Genetic association analysis of ERBB4 polymorphisms with the risk of schizophrenia and SPEM abnormality in a Korean population. Brain Res 1466:146-151. CrossRef Medline

Bao J, Wolpowitz D, Role LW, Talmage DA (2003) Back-signaling by the Nrg-1 intracellular domain. J Cell Biol 161:1133-1141. CrossRef Medline

Bao J, Lin H, Ouyang Y, Lei D, Osman A, Kim TW, Mei L, Dai P, Ohlemiller KK, Ambron RT (2004) Activity-dependent transcription regulation of PSD-95 by neuregulin-1 and Eos. Nat Neurosci 7:1250-1258. CrossRef Medline

Bjarnadottir M, Misner DL, Haverfield-Gross S, Bruun S, Helgason VG, Stefansson H, Sigmundsson A, Firth DR, Nielsen B, Stefansdottir R, Novak TJ, Stefansson K, Gurney ME, Andresson T (2007) Neuregulin1 (NRG1) signaling through Fyn modulates NMDA receptor phosphorylation: differential synaptic function in NRG1 ${ }^{+/-}$knock-outs compared with wild-type mice. J Neurosci 27:4519-4529. CrossRef Medline

Brinkmann BG, Agarwal A, Sereda MW, Garratt AN, Müller T, Wende H, Stassart RM, Nawaz S, Humml C, Velanac V, Radyushkin K, Goebbels S, Fischer TM, Franklin RJ, Lai C, Ehrenreich H, Birchmeier C, Schwab MH, Nave KA (2008) Neuregulin-1/ErbB signaling serves distinct functions in myelination of the peripheral and central nervous system. Neuron 59:581-595. CrossRef Medline

Buonanno A, Kwon OB, Yan L, Gonzalez C, Longart M, Hoffman D, Vullhorst D (2008) Neuregulins and neuronal plasticity: possible relevance in schizophrenia. Novartis Found Symp 289:165-177. CrossRef

Canetta SE, Luca E, Pertot E, Role LW, Talmage DA (2011) Type III Nrg1 back-signaling enhances functional TRPV1 along sensory axons contributing to basal and inflammatory thermal pain sensation. PloS One 6:e25108. CrossRef Medline

Carlsen J, Záborszky L, Heimer L (1985) Cholinergic projections from the basal forebrain to the basolateral amygdaloid complex: a combined retrograde fluorescent and immunohistochemical study. J Comp Neurol 234:155-167. CrossRef Medline

Chang Q, Fischbach GD (2006) An acute effect of neuregulin 1 beta to suppress $\alpha 7$-containing nicotinic acetylcholine receptors in hippocampal interneurons. J Neurosci 26:11295-11303. CrossRef Medline

Chen B, Wang SS, Hattox AM, Rayburn H, Nelson SB, McConnell SK (2008a) The Fezf2-Ctip2 genetic pathway regulates the fate choice of subcortical projection neurons in the developing cerebral cortex. Proc Natl Acad Sci U S A 105:11382-11387. CrossRef Medline

Chen YJ, Johnson MA, Lieberman MD, Goodchild RE, Schobel S, Lewandowski N, Rosoklija G, Liu RC, Gingrich JA, Small S, Moore H, Dwork AJ, Talmage DA, Role LW (2008b) Type III neuregulin-1 is required for normal sensorimotor gating, memory-related behaviors, and corticostriatal circuit components. J Neurosci 28:6872-6883. CrossRef Medline

Chen Y, Hancock ML, Role LW, Talmage DA (2010a) Intramembranous valine linked to schizophrenia is required for neuregulin 1 regulation of the morphological development of cortical neurons. J Neurosci 30:91999208. CrossRef Medline

Chen YJ, Zhang M, Yin DM, Wen L, Ting A, Wang P, Lu YS, Zhu XH, Li SJ, Wu CY, Wang XM, Lai C, Xiong WC, Mei L, Gao TM (2010b) ErbB4 in parvalbumin-positive interneurons is critical for neuregulin 1 regulation of long-term potentiation. Proc Natl Acad Sci U S A 107:21818-21823. CrossRef Medline

Ehrlich I, Humeau Y, Grenier F, Ciocchi S, Herry C, Lüthi A (2009) Amygdala inhibitory circuits and the control of fear memory. Neuron 62:757-771. CrossRef Medline

Fazzari P, Paternain AV, Valiente M, Pla R, Luján R, Lloyd K, Lerma J, Marín O, Rico B (2010) Control of cortical GABA circuitry development by Nrg1 and ErbB4 signalling. Nature 464:1376-1380. CrossRef Medline

Fenster C, Vullhorst D, Buonanno A (2012) Acute neuregulin-1 signaling influences AMPA receptor-mediated responses in cultured cerebellar granule neurons. Brain Res Bull 87:21-29. CrossRef Medline

Freedman R, Leonard S, Gault JM, Hopkins J, Cloninger CR, Kaufmann CA, Tsuang MT, Farone SV, Malaspina D, Svrakic DM, Sanders A, Gejman P (2001) Linkage disequilibrium for schizophrenia at the chromosome 15q13-14 locus of the $\alpha 7$-nicotinic acetylcholine receptor subunit gene (CHRNA7). Am J Med Genet 105:20-22. CrossRef Medline

Hancock ML, Canetta SE, Role LW, Talmage DA (2008) Presynaptic type III neuregulin1-ErbB signaling targets $\alpha 7$ nicotinic acetylcholine receptors to axons. J Cell Biol 181:511-521. CrossRef Medline

Hancock ML, Nowakowski DW, Role LW, Talmage DA, Flanagan JG (2011) Type III neuregulin 1 regulates pathfinding of sensory axons in the developing spinal cord and periphery. Development 138:4887-4898. CrossRef Medline

Harrison PJ, Law AJ (2006) Neuregulin 1 and schizophrenia: genetics, gene expression, and neurobiology. Biol Psychiatry 60:132-140. CrossRef Medline

Harrison PJ, Weinberger DR (2005) Schizophrenia genes, gene expression, and neuropathology: on the matter of their convergence. Mol Psychiatry 10:40-68. CrossRef Medline

Jiang L, Role LW (2008) Facilitation of cortico-amygdala synapses by nicotine: activity-dependent modulation of glutamatergic transmission. J Neurophysiol 99:1988-1999. CrossRef Medline

Kwon OB, Longart M, Vullhorst D, Hoffman DA, Buonanno A (2005) Neuregulin-1 reverses long-term potentiation at CA1 hippocampal synapses. J Neurosci 25:9378-9383. CrossRef Medline

Leonard S, Freedman R (2006) Genetics of chromosome 15q13-q14 in schizophrenia. Biol Psychiatry 60:115-122. CrossRef Medline

Li B, Woo RS, Mei L, Malinow R (2007) The neuregulin-1 receptor erbB4 controls glutamatergic synapse maturation and plasticity. Neuron 54: 583-597. CrossRef Medline

López-Bendito G, Cautinat A, Sánchez JA, Bielle F, Flames N, Garratt AN, Talmage DA, Role LW, Charnay P, Marín O, Garel S (2006) Tangential neuronal migration controls axon guidance: a role for neuregulin-1 in thalamocortical axon navigation. Cell 125:127-142. CrossRef Medline

Lu CL, Wang YC, Chen JY, Lai IC, Liou YJ (2010) Support for the involvement of the ERBB4 gene in schizophrenia: a genetic association analysis. Neurosci Lett 481:120-125. CrossRef Medline

Mathew SV, Law AJ, Lipska BK, Dávila-Garcia MI, Zamora ED, Mitkus SN, Vakkalanka R, Straub RE, Weinberger DR, Kleinman JE, Hyde TM (2007) Alpha7 nicotinic acetylcholine receptor mRNA expression and binding in postmortem human brain are associated with genetic variation in neuregulin 1. Hum Mol Genet 16:2921-2932. CrossRef Medline

McKenna WL, Betancourt J, Larkin KA, Abrams B, Guo C, Rubenstein JL, Chen B (2011) Tbr1 and Fezf2 regulate alternate corticofugal neuronal identities during neocortical development. J Neurosci 31:549-564. CrossRef Medline

Michailov GV, Sereda MW, Brinkmann BG, Fischer TM, Haug B, Birchmeier C, Role L, Lai C, Schwab MH, Nave KA (2004) Axonal neuregulin-1 regulates myelin sheath thickness. Science 304:700-703. CrossRef Medline

Molyneaux BJ, Arlotta P, Hirata T, Hibi M, Macklis JD (2005) Fezl is required for the birth and specification of corticospinal motor neurons. Neuron 47:817-831. CrossRef Medline

Muller JF, Mascagni F, McDonald AJ (2011) Cholinergic innervation of pyramidal cells and parvalbumin-immunoreactive interneurons in the rat basolateral amygdala. J Comp Neurol 519:790-805. CrossRef Medline

Neddens J, Vullhorst D, Paredes D, Buonanno A (2009) Neuregulin links dopaminergic and glutamatergic neurotransmission to control hippocampal synaptic plasticity. Commun Integr Biol 2:261-264. CrossRef Medline

Paxinos G, Franklin K (2004) The mouse brain in stereotaxic coordinates, Ed 2. Amsterdam: Elsevier.

Pitcher GM, Beggs S, Woo RS, Mei L, Salter MW (2008) ErbB4 is a suppressor of long-term potentiation in the adult hippocampus. Neuroreport 19:139-143. CrossRef Medline

Pitcher GM, Kalia LV, Ng D, Goodfellow NM, Yee KT, Lambe EK, Salter MW (2011) Schizophrenia susceptibility pathway neuregulin 1-ErbB4 suppresses Src upregulation of NMDA receptors. Nat Med 17:470-478. CrossRef Medline

Quintes S, Goebbels S, Saher G, Schwab MH, Nave KA (2010) Neuron-glia 
signaling and the protection of axon function by Schwann cells. J Peripher Nerv Syst 15:10-16. CrossRef Medline

Sandrock AW Jr, Dryer SE, Rosen KM, Gozani SN, Kramer R, Theill LE, Fischbach GD (1997) Maintenance of acetylcholine receptor number by neuregulins at the neuromuscular junction in vivo. Science 276:599-603. CrossRef Medline

Schäfer MK, Eiden LE, Weihe E (1998) Cholinergic neurons and terminal fields revealed by immunohistochemistry for the vesicular acetylcholine transporter: I. Central nervous system. Neuroscience 84:331-359. CrossRef Medline

Shamir A, Kwon OB, Karavanova I, Vullhorst D, Leiva-Salcedo E, Janssen MJ, Buonanno A (2012) The importance of the NRG-1/ErbB4 pathway for synaptic plasticity and behaviors associated with psychiatric disorders. J Neurosci 32:2988-2997. CrossRef Medline

Stefanis NC, Hatzimanolis A, Smyrnis N, Avramopoulos D, Evdokimidis I, van Os J, Stefanis CN, Straub RE, Weinberger DR (2013) Schizophrenia candidate gene ERBB4: covert routes of vulnerability to psychosis detected at the population level. Schizophr Bull 39:349-357. CrossRef Medline

Stefansson H, Sigurdsson E, Steinthorsdottir V, Bjornsdottir S, Sigmundsson T, Ghosh S, Brynjolfsson J, Gunnarsdottir S, Ivarsson O, Chou TT, Hjaltason O, Birgisdottir B, Jonsson H, Gudnadottir VG, Gudmundsdottir E, Bjornsson A, Ingvarsson B, Ingason A, Sigfusson S, Hardardottir H, et al. (2002) Neuregulin 1 and susceptibility to schizophrenia. Am J Hum Genet 71:877-892. CrossRef Medline

Taveggia C, Zanazzi G, Petrylak A, Yano H, Rosenbluth J, Einheber S, Xu X,
Esper RM, Loeb JA, Shrager P, Chao MV, Falls DL, Role L, Salzer JL (2005) Neuregulin-1 type III determines the ensheathment fate of axons. Neuron 47:681-694. CrossRef Medline

Ting AK, Chen Y, Wen L, Yin DM, Shen C, Tao Y, Liu X, Xiong WC, Mei L (2011) Neuregulin 1 promotes excitatory synapse development and function in GABAergic interneurons. J Neurosci 31:15-25. CrossRef Medline

Tsvetkov E, Shin RM, Bolshakov VY (2004) Glutamate uptake determines pathway specificity of long-term potentiation in the neural circuitry of fear conditioning. Neuron 41:139-151. CrossRef Medline

Wenk GL (1997) The nucleus basalis magnocellularis cholinergic system: one hundred years of progress. Neurobiol Learn Mem 67:85-95. CrossRef Medline

Wolpowitz D, Mason TB, Dietrich P, Mendelsohn M, Talmage DA, Role LW (2000) Cysteine-rich domain isoforms of the neuregulin-1 gene are required for maintenance of peripheral synapses. Neuron 25:79-91. CrossRef Medline

Yang X, Kuo Y, Devay P, Yu C, Role L (1998) A cysteine-rich isoform of neuregulin controls the level of expression of neuronal nicotinic receptor channels during synaptogenesis. Neuron 20:255-270. CrossRef Medline

Zhong C, Du C, Hancock M, Mertz M, Talmage DA, Role LW (2008) Presynaptic type III neuregulin 1 is required for sustained enhancement of hippocampal transmission by nicotine and for axonal targeting of $\alpha 7$ nicotinic acetylcholine receptors. J Neurosci 28:9111-9116. CrossRef Medline 